TRANSACTIONS OF THE

AMERICAN MATHEMATICAL SOCIETY

Volume 354, Number 2, Pages 807-836

S 0002-9947(01)02785-4

Article electronically published on September 19, 2001

\title{
INDUCED OPERATORS ON SYMMETRY CLASSES OF TENSORS
}

\author{
CHI-KWONG LI AND ALEXANDRU ZAHARIA
}

\begin{abstract}
Let $V$ be an $n$-dimensional Hilbert space. Suppose $H$ is a subgroup of the symmetric group of degree $m$, and $\chi: H \rightarrow \mathbb{C}$ is a character of degree 1 on $H$. Consider the symmetrizer on the tensor space $\bigotimes^{m} V$

$$
S\left(v_{1} \otimes \cdots \otimes v_{m}\right)=\frac{1}{|H|} \sum_{\sigma \in H} \chi(\sigma) v_{\sigma^{-1}(1)} \otimes \cdots \otimes v_{\sigma^{-1}(m)}
$$

defined by $H$ and $\chi$. The vector space

$$
V_{\chi}^{m}(H)=S(\stackrel{m}{\bigotimes} V)
$$

is a subspace of $\otimes^{m} V$, called the symmetry class of tensors over $V$ associated with $H$ and $\chi$. The elements in $V_{\chi}^{m}(H)$ of the form $S\left(v_{1} \otimes \cdots \otimes v_{m}\right)$ are called decomposable tensors and are denoted by $v_{1} * \cdots * v_{m}$. For any linear operator $T$ acting on $V$, there is a (unique) induced operator $K(T)$ acting on $V_{\chi}^{m}(H)$ satisfying

$$
K(T) v_{1} * \cdots * v_{m}=T v_{1} * \cdots * T v_{m} .
$$
\end{abstract}

In this paper, several basic problems on induced operators are studied.

In this paper, we study induced operator $K(T)$ acting on symmetry classes of tensors. In Chapter 1, some background and notations are presented.

It is known that if $T$ is normal, unitary, positive (semi-)definite, (skew) hermitian, then $K(T)$ has the corresponding property. Furthermore, if $T_{1}=\xi T_{2}$ for some $\xi \in \mathbb{C}$ with $\xi^{m}=1$, then $K\left(T_{1}\right)=K\left(T_{2}\right)$. However, the converse of these statements are not valid in general. Counterexamples have been given and special instances under which the converses hold have been identified in the literature. In Chapter 2, necessary and sufficient conditions on $\chi$ and the operators $T, T_{1}, T_{2}$ ensuring the validity of the converses of the above statements are given. These results explain the counterexamples and special results obtained by other researchers.

The decomposable numerical range $W_{\chi}(T)$ of $T$ is the set of complex numbers of the form $\left(K(T) x^{*}, x^{*}\right)$ with $x^{*}$ ranging over all decomposable unit vectors in $V_{\chi}^{m}(H)$, and the decomposable numerical radius $r_{\chi}(T)$ of $T$ is the radius of the smallest circular disk in $\mathbb{C}$ centered at the origin including $W_{\chi}(T)$. Chapter 3 is devoted to the study of these two concepts. Relations between geometric properties of $W_{\chi}(T)$ and algebraic properties of $T$ are explored. Matrix inequalities involving the decomposable numerical radius $r_{\chi}(T)$, the spectral norm $\|K(T)\|$, and the spectral radius $\rho(K(T))$ are investigated; matrices attaining the equalities are characterized.

Received by the editors October 6, 1999 and, in revised form, September 11, 2000.

2000 Mathematics Subject Classification. Primary 15A69, 15A60, 15A42, 15A45, 15A04, $47 \mathrm{~B} 49$.

Key words and phrases. Symmetry class of tensors, linear operator, induced operator. 
In Chapter 4, some invariance problems (also known as the linear preserver or transformer problems) of functions $F(T)$ related to the induced operators $T$, such as $F(T)=\|K(T)\|, \rho(K(T)), r_{\chi}(T), W_{\chi}(T)$, are studied. In particular, the structure of those linear operators $L$ on $\operatorname{End}(V)$, the algebra of endomorphisms of $V$, satisfying $F(T)=F(L(T))$ for all $T \in \operatorname{End}(V)$ are determined for various functions $F$ including those mentioned above. In many cases, the linear operators $L$ are algebra isomorphisms or anti-isomorphisms (possibly followed by the multiplication of unitary operators) on $\operatorname{End}(V)$.

Our results settle a number of open problems and extend many results of other researchers on the subject.

\section{BACKGROUND AND Notations}

Let $V$ be an $n$-dimensional Hilbert space. Suppose $H$ is a subgroup of the symmetric group of degree $m$, and $\chi: H \rightarrow \mathbb{C}$ is a character of degree 1 on $H$. Consider the symmetrizer on the tensor space $\otimes^{m} V$

$$
S\left(v_{1} \otimes \cdots \otimes v_{m}\right)=\frac{1}{|H|} \sum_{\sigma \in H} \chi(\sigma) v_{\sigma^{-1}(1)} \otimes \cdots \otimes v_{\sigma^{-1}(m)}
$$

defined by $H$ and $\chi$. The vector space

$$
V_{\chi}^{m}(H)=S\left(\bigotimes^{m} V\right)
$$

is a subspace of $\bigotimes^{m} V$, called the symmetry class of tensors over $V$ associated with $H$ and $\chi$. The elements in $V_{\chi}^{m}(H)$ of the form $S\left(v_{1} \otimes \cdots \otimes v_{m}\right)$ are called decomposable tensors and are denoted by $v_{1} * \cdots * v_{m}$.

The study of symmetry classes of tensors is motivated by many branches of pure and applied mathematics: combinatorial theory, matrix theory, operator theory, group representation theory, differential geometry, partial differential equations, quantum mechanics and other areas. See [18, 36, 37, 45] for some general background.

For any linear operator $T$ acting on $V$, there is a (unique) induced operator $K(T)$ acting on $V_{\chi}^{m}(H)$ satisfying

$$
K(T) v_{1} * \cdots * v_{m}=T v_{1} * \cdots * T v_{m} .
$$

The induced operator is a useful object in the study of symmetry classes of tensors. In this paper, we study some basic problems concerning induced operators.

Let $M_{m}$ be the set of $m \times m$ complex matrices. Define the generalized matrix function $d_{\chi}: M_{m} \rightarrow \mathbb{C}$ associated with $\chi$ by

$$
d_{\chi}(X)=\sum_{\sigma \in H} \chi(\sigma) \prod_{j=1}^{m} X_{j, \sigma(j)}, \quad X=\left(X_{j k}\right) \in M_{m} .
$$

One can use this concept to facilitate the study of symmetry classes of tensors and induced operators. For instance, the inner product on $V_{\chi}^{m}(H)$ can be expressed in terms of the inner product $(u, v)$ on $V$ by

$$
\left(u_{1} * \cdots * u_{m}, v_{1} * \cdots * v_{m}\right)=\frac{1}{|H|} d_{\chi}\left[\left(u_{i}, v_{j}\right)\right] .
$$

In fact, if we identify $V$ with $\mathbb{C}^{n}$ with respect to a fixed orthonormal basis $\mathcal{B}$, then a decomposable tensor $v^{*}=v_{1} * \cdots * v_{m} \in V_{\chi}^{m}(H)$ can be identified with the 
$n \times m$ matrix $X$ such that the $j$ th column of $X$ is the coordinate vector of $v_{j}$ with respect to $\mathcal{B}$ and

$$
\left(v^{*}, v^{*}\right)=\frac{1}{|H|} d_{\chi}\left(X^{*} X\right) .
$$

Furthermore, one can generate an orthonormal basis for $V_{\chi}^{m}(H)$ from one of $V$, and exhibit a matrix representation of an induced operator $K(T)$. We need some more notation to do that.

Let $\Gamma_{m, n}$ be the set of sequences $\alpha=(\alpha(1), \ldots, \alpha(m))$ with $1 \leq \alpha(j) \leq n$ for $j=1, \ldots, m$. For $r=1, \ldots, n$ and $\alpha \in \Gamma_{m, n}$, let $m_{r}(\alpha)$ be the number of times the integer $r$ appears in $\alpha$. Two sequences $\alpha$ and $\beta$ in $\Gamma_{m, n}$ are said to be equivalent modulo $H$, denoted by $\alpha \sim \beta$, if there exists $\sigma \in H$ such that $\beta=\alpha \sigma$. Evidently, this equivalence relation partitions $\Gamma_{m, n}$ into equivalence classes. Let $\Delta$ be a system of representatives for the equivalence classes so that each sequence in $\Delta$ is first in lexicographic order in its equivalence class. Define $\bar{\Delta}$ as the subset of $\Delta$ consisting of those sequences $w \in \Delta$ such that

$$
\nu(w)=\sum_{\sigma \in H_{w}} \chi(\sigma) \neq 0,
$$

where $H_{w}$ is the stabilizer of $w$, i.e., $H_{w}=\{\sigma \in H: w \sigma=w\}$.

Now, suppose $\mathcal{B}=\left\{e_{1}, \ldots, e_{n}\right\}$ is an orthonormal basis for $V$. Then

$$
\left\{e_{\alpha(1)} * \cdots * e_{\alpha(m)}: \alpha \in \bar{\Delta}\right\}
$$

is an orthogonal basis for $V_{\chi}^{m}(H)$, and one can get an orthonormal basis $\tilde{\mathcal{B}}$ after normalization. Furthermore, for any $A=\left(a_{j k}\right) \in M_{n}$, denote by $A[\beta \mid \alpha]$ the $m \times m$ matrix with $(r, s)$ entry equal to $a_{\beta(r), \alpha(s)}$. If $A \in M_{n}$ is the matrix representation of $T$ with respect to $\mathcal{B}$, then the induced operator $K(T)$ has a matrix representation with respect to the basis $\tilde{\mathcal{B}}$, denoted by $K(A)$. In fact (see e.g. [36, p. 126]), $K(A)$ is an $|\bar{\Delta}| \times|\bar{\Delta}|$ matrix with rows and columns indexed lexicographically by the set $\bar{\Delta}$ so that the entry of $K(A)$ labeled by $(\alpha, \beta)$ in $\bar{\Delta} \times \bar{\Delta}$ is equal to

$$
\frac{1}{\sqrt{\nu(\alpha) \nu(\beta)}} d_{\chi}\left(A^{t}[\beta \mid \alpha]\right) \text {. }
$$

By the above discussion, we can identify $V$ with $\mathbb{C}^{n}, T$ with $A \in M_{n}$ and $K(T)$ with $K(A)$, etc. In particular, the induced matrix $K(A)$ is the linear operator acting on the decomposable tensor of $V_{\chi}^{m}(H)$ according to the formula

$$
K(A) x_{1} * \cdots * x_{m}=A x_{1} * \cdots * A x_{m}, \quad x_{1}, \ldots, x_{m} \in \mathbb{C}^{n} .
$$

From this point, we shall work on these matrix formulations of the induced operators. Furthermore, we shall always assume that $\bar{\Delta} \neq \emptyset$ so that $K(A)$ is well-defined.

We give several common examples of symmetry classes of tensors and induced operators in the following.

Example 1.1. Let $1 \leq m \leq n, H=S_{m}$ and $\chi$ be the alternate character. Then $V_{\chi}^{m}(H)$ is the $m$ th exterior space over $V=\mathbb{C}^{n}, \bar{\Delta}$ is the set of strictly increasing sequences in $\Gamma_{m, n}, d_{\chi}(B)=\operatorname{det}(B)$ is the determinant for $B \in M_{m}$, and $K(A)$ is the $m$ th compound matrix of $A \in M_{n}$.

Example 1.2. Let $H=S_{m}$ and $\chi=1$ be the principal character. Then $V_{\chi}^{m}(H)$ is the $m$ th completely symmetric space over $V=\mathbb{C}^{n}, \bar{\Delta}=\Gamma_{m, n}, d_{\chi}(B)=\operatorname{per}(B)$ is the permanent for $B \in M_{m}$, and $K(A)$ is the $m$ th induced power of $A \in M_{n}$. 
Example 1.3. Let $H=\{e\}$ where $e$ is the identity in $S_{m}$ and let $\chi=1$ be the principal character. Then $V_{\chi}^{m}(H)=\bigotimes^{m} V, \bar{\Delta}=\Gamma_{m, n}, d_{\chi}(B)=\prod_{j=1}^{m} b_{j j}$ for $B=\left(b_{j k}\right) \in M_{m}$, and $K(A)=\bigotimes^{k} A$ is the $m$ th tensor power of $A \in M_{n}$.

We list some basic properties of $K(A)$ in the following (see [36, Chapter 2]).

Proposition 1.4. The following properties hold for induced matrices:

(a) $K\left(I_{n}\right)=I_{|\bar{\Delta}|}$.

(b) $K(A B)=K(A) K(B)$ for any $A, B \in M_{n}$.

(c) $K\left(A^{*}\right)=K(A)^{*}$ for any $A \in M_{n}$.

(d) $A \in M_{n}$ is invertible if and only if $K(A)$ is. Moreover, we have $K\left(A^{-1}\right)=$ $K(A)^{-1}$.

(e) If $A \in M_{n}$ is in (lower or upper) triangular or in diagonal form, then so is $K(A)$.

(f) If $A$ has eigenvalues $\lambda_{1}, \ldots, \lambda_{n}$, and singular values $s_{1} \geq \cdots \geq s_{n}$, then for any $\sigma \in S_{n}, K(A)$ has eigenvalues $\prod_{j=1}^{n} \lambda_{\sigma(j)}^{m_{j}(\alpha)}$ and singular values $\prod_{j=1}^{n} s_{\sigma(j)}^{m_{j}(\alpha)}$, $\alpha \in \bar{\Delta}$.

(g) $\operatorname{det}(K(A))=\operatorname{det}(A)^{k}$, where $k=|\bar{\Delta}| m / n$.

(h) If $\operatorname{rank}(A)=r$, then $\operatorname{rank}(K(A))=\left|\Gamma_{m, r} \cap \bar{\Delta}\right|$.

Note that part (f) in the above proposition is usually stated with $\sigma$ equal to the identity permutation. In our statement, we emphasis that relabeling of the indices of the eigenvalues or singular values will not affect the conclusion. This observation will be used frequently in our study.

In the subsequent discussion, we shall use $\left\{E_{11}, E_{12}, \ldots, E_{n n}\right\}$ to denote the standard basis for $M_{n}$, and we use $\mu(\bar{\Delta})$ to denote the smallest integer $r$ such that $\Gamma_{m, r} \cap \bar{\Delta} \neq \emptyset$. As a result, a matrix $A \in M_{n}$ satisfies $K(A)=0$ if and only if $\operatorname{rank}(A)<\mu(\bar{\Delta})$.

\section{Normality and Equality of Induced Operators}

Using Proposition 1.4 one easily obtains the following result (see [36, Chapter 2]).

Proposition 2.1. Let $A \in M_{n}$. If $A$ is normal, unitary, positive (semi-)definite, hermitian or skew-hermitian (when $m$ is odd), then $K(A)$ has the corresponding property.

It is natural to ask whether the converse of the above proposition holds. Unfortunately, it is not true in general as noted in [36] p. 148]. For example, if $1<m \leq n$ and if $A \in M_{n}$ has $\operatorname{rank}(A)<m$, then $K(A)=0$ is trivially normal. Of course, not much can be said about such a matrix $A$.

Even if $K(A)$ is nonzero, the converse of Proposition 2.1] may fail. For instance, if $\chi$ is the alternate character and $m=n$, then $K(A)=(\operatorname{det}(A))$ is always normal, and one may impose a suitable condition on $\operatorname{det}(A)$ to ensure $K(A)$ to be unitary, (skew-)hermitian or positive (semi-)definite.

If $\chi$ is the alternate character and $m<n$, then $V_{\chi}^{m}(H)$ has dimension $\left(\begin{array}{c}n \\ m\end{array}\right)$. Suppose $A=A_{1} \oplus 0_{n-m}$ such that $A_{1} \in M_{m}$ is not normal. Then $K(A)=$ $\operatorname{diag}\left(\operatorname{det}\left(A_{1}\right), 0, \ldots, 0\right)$ is normal, and again one may impose a suitable condition on $\operatorname{det}\left(A_{1}\right)$ to ensure $K(A)$ to be nonzero, (skew-)hermitian or positive semi-definite.

Despite the negative examples mentioned in the last few paragraphs, the converse of Proposition 2.1 indeed holds under certain assumptions on $\chi$ or $A$. For example, 
by Ex. 28 and 29 in p. 157, Theorem 4.7 in Chapter 2 of [36], if $\chi$ is the principal character or if $\operatorname{rank}(A)>m$, then the following hold.

(I) If $K(A) \neq 0$ is normal or unitary, then $A$ has the corresponding property.

(II) If there exists $\eta \in \mathbb{C}$ with $|\eta|=1$ such that $\eta K(A) \neq 0$ is hermitian or positive (semi-)definite, then $\xi A$ also has the corresponding property for some $\xi \in \mathbb{C}$ with $\xi^{m}=\eta$.

Since $K(A)=K(\xi A)$ if $\xi \in \mathbb{C}$ satisfies $\xi^{m}=1$, we see that (II) is the reasonable statement to pursue. In fact, the proofs in [36] were done based on the fact that if $\chi$ is the principal character or if $\operatorname{rank}(A)>m$, then the following holds.

(III) A matrix $B \in M_{n}$ satisfies $K(A)=K(B)$ if and only if $B=\xi A$ for some $\xi \in \mathbb{C}$ with $\xi^{m}=1$.

Again, examples were given to show that the hypotheses on the character $\chi$ and the matrix $A$ are needed.

The purpose of this chapter is to determine the necessary and sufficient conditions on $A$ and $\chi$ so that (I), (II) or (III) holds. In addition to their own interest, the results are useful for the study on quadratic forms, decomposable numerical ranges and radii, inequalities, etc., involving induced operators as shown in later chapters.

We present some preliminary lemmas in $\S 2.1$. Then we identify special types of characters in $\S 2.2$ for which (I)-(III) may fail. Complete solutions of our problems in connections with (I)-(III) will be given in $\S 2.3$.

2.1. Preliminary Lemmas. The first lemma is due to Horn [22] and Weyl [57].

Lemma 2.2. Suppose $\lambda_{1}, \ldots, \lambda_{n}$ are complex numbers with $\left|\lambda_{1}\right| \geq \cdots \geq\left|\lambda_{n}\right|$, and $s_{1} \geq \cdots \geq s_{n}$ are nonnegative real numbers. Then there exists $A \in M_{n}$ with singular values $s_{1}, \ldots, s_{n}$ and eigenvalues $\lambda_{1}, \ldots, \lambda_{n}$ if and only if $\prod_{j=1}^{n}\left|\lambda_{j}\right|=\prod_{j=1}^{n} s_{j}$ and

$$
\prod_{j=1}^{k}\left|\lambda_{j}\right| \leq \prod_{j=1}^{k} s_{j} \quad \text { for } k=1, \ldots, n-1 .
$$

The next lemma can be found in [30].

Lemma 2.3. Let $A \in M_{n}$ have singular values $s_{1} \geq \cdots \geq s_{n}$ and eigenvalues $\lambda_{1}, \ldots, \lambda_{n}$ with $\left|\lambda_{1}\right| \geq \cdots \geq\left|\lambda_{n}\right|$. Suppose $1 \leq k \leq n$.

(a) If $\prod_{j=1}^{k} s_{j}=\prod_{j=1}^{k}\left|\lambda_{j}\right|>0$, then $A$ is unitarily similar to $A_{1} \oplus A_{2}$ with $A_{1} \in M_{k}$ so that $\left|\operatorname{det}\left(A_{1}\right)\right|=\prod_{j=1}^{k} s_{j}$.

(b) We have $\sum_{j=1}^{k}\left|\lambda_{j}\right| \leq \sum_{j=1}^{k} s_{j}$. The equality holds if and only if $A$ is unitarily similar to $\operatorname{diag}\left(\lambda_{1}, \ldots, \lambda_{k}\right) \oplus A_{2}$, where $\left|\lambda_{j}\right|=s_{j}$ for $j=1, \ldots, k$.

(c) There exists $k \geq n-1$ such that $\sum_{j=1}^{k}\left|\lambda_{j}\right|=\sum_{j=1}^{k} s_{j}$ if and only if $A$ is normal.

Lemma 2.4. Suppose $A, B \in M_{n}$ are positive semi-definite matrices with eigenvalues $a_{1} \geq \cdots \geq a_{n} \geq 0$ and $b_{1} \geq \cdots \geq b_{n} \geq 0$, respectively. Suppose $\prod_{j=1}^{k} a_{j} \leq$ $\prod_{j=1}^{k} b_{j}$ for all $k=1, \ldots, n$. Then

$$
\operatorname{tr} K(A) \leq \operatorname{tr} K(B) .
$$

Proof. Suppose $A$ and $B$ satisfy the assumption. By Lemma 2.2, there exists a complex matrix $C$ with singular values $b_{1} \geq \cdots \geq b_{n-1} \geq \tilde{b}_{n}$ and eigenvalues 
$a_{1} \geq \cdots \geq a_{n}$, where

$$
\tilde{b}_{n}= \begin{cases}\left(a_{1} \ldots a_{n}\right) /\left(b_{1} \cdots b_{n-1}\right) & \text { if } b_{n}>0 \\ 0 & \text { otherwise. }\end{cases}
$$

By Lemma 2.3 and Proposition 1.4 (f), we have

$$
\operatorname{tr} K(A)=\operatorname{tr} K(C) \leq \operatorname{tr}\left\{K(C)^{*} K(C)\right\}^{1 / 2} \leq \operatorname{tr} K(B) .
$$

Lemma 2.5. Suppose $\Gamma_{m, r} \cap \bar{\Delta}$ contains an element $\alpha$ with $m_{p}(\alpha)>m_{q}(\alpha)$ for some $1 \leq p<q \leq r$. Let $A=\operatorname{diag}\left(a_{1}, \ldots, a_{r}, 0, \ldots, 0\right)$ with $a_{1} \geq \cdots \geq a_{r}>0$. Suppose $B=\operatorname{diag}\left(b_{1}, \ldots, b_{r}, 0, \ldots, 0\right)$ is obtained from $A$ by replacing $\left(a_{j}, a_{j+1}\right)$ with $\left(a_{j} t, a_{j+1} / t\right)$ for some $t>1$, where $1 \leq j<r$. Then $\operatorname{tr} K(A)<\operatorname{tr} K(B)$.

Proof. By Proposition 1.4(f), the mapping

$$
\left(x_{1}, \ldots, x_{n}\right) \mapsto \operatorname{tr} K\left(\operatorname{diag}\left(x_{1}, \ldots, x_{n}\right)\right)
$$

is a symmetric polynomial function in $x_{1}, \ldots, x_{n}$. Thus $\operatorname{tr} K(B)-\operatorname{tr} K(A)$ is a nonnegative combination of terms of the form

$$
\left(a_{j} t\right)^{k}+\left(a_{j+1} / t\right)^{k}-\left(a_{j}^{k}+a_{j+1}^{k}\right)
$$

for some $k \in\{1, \ldots, r\}$. By elementary calculus, one easily checks that the expression in (1) is positive. Since $\Gamma_{m, r} \cap \bar{\Delta}$ contains an element $\alpha$ with $m_{p}(\alpha)>m_{q}(\alpha)$ for some $p \neq q$, there exists $\beta \in \Gamma_{m, r} \cap \bar{\Delta}$ such that $m_{j}(\beta)>m_{j+1}(\beta)$. Furthermore, we can obtain $\gamma \in \Gamma_{m, r} \cap \bar{\Delta}$ such that $\left(m_{j}(\gamma), m_{j+1}(\gamma)\right)=\left(m_{j+1}(\beta), m_{j}(\beta)\right)$, and $m_{t}(\gamma)=m_{t}(\beta)$ for $t \neq j, j+1$. Thus, we see that the coefficient of $\left(a_{j} t\right)^{k}+$ $\left(a_{j+1} / t\right)^{k}-\left(a_{j}^{k}+a_{j+1}^{k}\right)$ is nonzero for $k=m_{j}(\beta)-m_{j+1}(\beta)$. As a result, we have $\operatorname{tr} K(A)<\operatorname{tr} K(B)$.

2.2. Different Types of Characters. In the following, we identify different types of character $\chi$ so that (I)-(III) fail. Recall that $\mu(\bar{\Delta})$ is the smallest integer $r$ such that $\Gamma_{m, r} \cap \bar{\Delta}$ is nonempty.

Theorem 2.6. Let $\tilde{r}$ be an integer satisfying $\tilde{r} \geq \mu(\bar{\Delta})>1$. The following conditions are equivalent.

(a) Every $\alpha \in \Gamma_{m, \tilde{r}} \cap \bar{\Delta}$ satisfies $m_{1}(\alpha)=\cdots=m_{\tilde{r}}(\alpha)$ and $\tilde{r}=\mu(\bar{\Delta})$.

(b) There exists a nonnormal $A \in M_{n}$ with $\operatorname{rank}(A)=\tilde{r}$ such that $K(A)$ is a normal matrix.

(c) If $A \in M_{n}$ is unitarily similar to $A_{1} \oplus 0_{n-\tilde{r}}$, where $A_{1} \in M_{\tilde{r}}$ is invertible, then $K(A)$ is a multiple of an (hermitian) orthogonal projection.

Proof. Let $r=\mu(\bar{\Delta})$. The implication (c) $\Longrightarrow$ (b) is clear.

(b) $\Longrightarrow$ (a): Suppose $A \in M_{n}$ is nonnormal and $\operatorname{rank}(A)=\tilde{r}$ so that $K(A)$ is a (nonzero) normal matrix. Assume that not every $\alpha \in \Gamma_{m, \tilde{r}} \cap \bar{\Delta}$ satisfies $m_{1}(\alpha)=$ $\cdots=m_{\tilde{r}}(\alpha)$. Let $A$ have singular values $s_{1} \geq \cdots \geq s_{n}$ and eigenvalues $\lambda_{1}, \ldots, \lambda_{n}$ with $\left|\lambda_{1}\right| \geq \cdots \geq\left|\lambda_{n}\right|$. As $A$ is not normal and has rank $\tilde{r}$, there is a smallest integer $p$ with $p \leq \tilde{r}$ such that $s_{p}>\left|\lambda_{p}\right|$. By Lemma 2.2, $\prod_{j=1}^{k}\left|\lambda_{j}\right| \leq \prod_{j=1}^{k} s_{j}$ for $k=1, \ldots, \tilde{r}$, and $\left|\prod_{j=1}^{n} \lambda_{j}\right|=\prod_{j=1}^{n} s_{j}$. Let $\tilde{D}=\operatorname{diag}\left(\left|\lambda_{1}\right|, \ldots,\left|\lambda_{n}\right|\right)$ and construct

$$
D=\operatorname{diag}\left(d_{1}, \ldots, d_{n}\right)
$$

as follows. If $s_{p}=\cdots=s_{\tilde{r}}$, set $d_{\tilde{r}}=\left|\lambda_{p}\right|$ and $d_{k}=s_{k}$ for other $k$. (Note that this can only happen when $\tilde{r}<n$.) If $s_{p}=\cdots=s_{j}>s_{j+1}$ for some $j<\tilde{r}$, set 
$t=\min \left\{s_{j} /\left|\lambda_{j}\right|, \sqrt{s_{j} / s_{j+1}}\right\}>1,\left(d_{j}, d_{j+1}\right)=\left(s_{j} / t, t s_{j+1}\right)$ and $d_{k}=s_{k}$ for other $k$. In both cases, we have $d_{1} \geq \cdots \geq d_{n}$ and

$$
\prod_{j=1}^{k}\left|\lambda_{j}\right| \leq \prod_{j=1}^{k} d_{j} \quad \text { for } k=1, \ldots, n-1,
$$

and $\prod_{j=1}^{n}\left|\lambda_{j}\right|=\prod_{j=1}^{n} d_{j}$, which is equal to 0 if $\tilde{r}<n$. By Lemma 2.4 we have $\operatorname{tr} K(\tilde{D}) \leq \operatorname{tr} K(D)$. By the definition of $D$ and Lemma 2.5, we have $\operatorname{tr} K(D)<$ $\operatorname{tr} K\left(\sum_{j=1}^{\tilde{r}} s_{j} E_{j j}\right)$. As a result, $\operatorname{tr} K(\tilde{D})<\operatorname{tr} K\left(\sum_{j=1}^{\tilde{r}} s_{j} E_{j j}\right)$. Since the eigenvalues of $K(\tilde{D})$ are just the moduli of those of $K(A)$, we have

$$
\sum_{\alpha \in \bar{\Delta}} \prod_{j=1}^{n}\left|\lambda_{j}^{m_{j}(\alpha)}\right|<\sum_{\alpha \in \bar{\Delta}} \prod_{j=1}^{n} s_{j}^{m_{j}(\alpha)} .
$$

By Lemma 2.3 $K(A)$ is not normal, which is a contradiction.

Finally, since $\Gamma_{m, r} \cap \bar{\Delta} \subseteq \Gamma_{m, \tilde{r}} \cap \bar{\Delta}$, if $\tilde{r}>r$, every element $\alpha \in \Gamma_{m, \tilde{r}} \cap \bar{\Delta}$ satisfies $m_{1}(\alpha)=\cdots=m_{r}(\alpha)=m_{\tilde{r}}(\alpha)=0$, which is a contradiction. Thus $\tilde{r}=r$.

(a) $\Longrightarrow(\mathrm{c})$ : Suppose (a) holds, and suppose $A$ is unitarily similar to $A_{1} \oplus 0_{n-r}$ such that $A_{1} \in M_{\tilde{r}}$ is invertible. Then the number of nonzero eigenvalues of $K(A)$ is the same as the number of the nonzero singular values of $K(A)$; all the nonzero eigenvalues and singular values have magnitude equal to $\left|\operatorname{det}\left(A_{1}\right)\right|^{m / r}$. Thus $K(A)$ is a multiple of an orthogonal projection.

Applying Theorem 2.6 with $\tilde{r}=n$, we get the following corollary (cf. Theorem 1.1 in [37, Chapter 6]).

Corollary 2.7. Let $H$ and $\chi$ be given. The following conditions are equivalent:

(a) Every element $\alpha \in \bar{\Delta}$ satisfies $m_{1}(\alpha)=\cdots=m_{n}(\alpha)=m / n$.

(b) $K(A)=(\operatorname{det} A)^{m / n} I$ for any $A \in M_{n}$.

(c) $K(A)$ is a scalar for any $A \in M_{n}$.

Definition 2.8. In the following, we say that $\chi$ is of determinant type if any one of the conditions (a)-(c) in Corollary 2.7 holds. Furthermore, we say that $\chi$ is of the special type if any one (and hence all) the conditions (a)-(c) in Theorem 2.6 hold with $\mu(\bar{\Delta})>1$; otherwise, we say that $\chi$ is of the general type.

Note that the alternate character on $S_{n}$ is of the determinant type; the alternate character on $S_{m}$ with $m<n$ is of the special type but not of the determinant type; and the principal character is of the general type. Here we give some additional examples of $\chi$ that are of the special type and the determinant type.

Example 2.9. Consider the alternating group in $S_{4}$ and use the character $\chi_{2}$ in [25, p. 181], i.e., $\chi_{2}(\sigma)=1$ if $\sigma$ is the identity permutation or a product of two disjoint transpositions, and if $1 \leq i<j<k \leq 4$, then $\chi_{2}((i, j, k))=w$ and $\chi_{2}((i, k, j))=w^{2}$, where $w=e^{2 \pi i / 3}$.

(a) If $n=2$, then

$$
\bar{\Delta}=\{(1,1,2,2)\}
$$

and $\chi_{2}$ is of the determinant type.

(b) If $n=3$, then

$$
\bar{\Delta}=\{(j, j, k, k): 1 \leq j<k \leq 3\} \cup\{(1,1,2,3),(1,2,2,3),(1,2,3,3)\},
$$

and $\chi_{2}$ is of the special type but not of the determinant type. 
2.3. Normality and Equality. Now, we can characterize $A \in M_{n}$ so that $K(A)$ is normal. We exclude the trivial case when $K(A)=0$, equivalently, when $A \in M_{n}$ has $\operatorname{rank}(A)<\mu(\bar{\Delta})$.

Theorem 2.10. Let $r=\mu(\bar{\Delta})$ and $A \in M_{n}$ with $\operatorname{rank}(A) \geq r$. Then $K(A)$ is a normal matrix if and only if one of the following holds.

(a) $A$ is normal.

(b) $\chi$ is of the special type, and $A$ is unitarily similar to $A_{1} \oplus 0_{n-r}$ such that $A_{1} \in M_{r}$ is an invertible nonnormal matrix.

Proof. If (a) holds, then $K(A)$ is normal by Proposition 2.1 If (b) holds, then $K(A)$ is normal by Theorem 2.6 (c).

Conversely, let $A \in M_{n}$ satisfy $\operatorname{rank}(A) \geq r$ so that $K(A)$ is a nonzero normal matrix. Assume that (a) does not hold. By Theorem 2.6, we see that $\operatorname{rank}(A)=r$ and $\chi$ is of the special type. Thus $A$ is unitarily similar to a block matrix of the form

$$
\left(\begin{array}{cc}
A_{1} & A_{2} \\
0 & 0_{n-r}
\end{array}\right)
$$

Since $K(A)$ is normal, all the nonzero eigenvalues $\left(\lambda_{1} \cdots \lambda_{r}\right)^{m / r}=\operatorname{det}\left(A_{1}\right)^{m / r}$ and all the nonzero singular values $\left(s_{1} \cdots s_{r}\right)^{m / r}=\left|\operatorname{det}\left(A_{1}\right)\right|^{m / r}$ have the same magnitude. Consequently, we have $s_{1} \cdots s_{r}=\left|\operatorname{det}\left(A_{1}\right)\right|$ and hence $A_{2}=0$ by Lemma 2.3 (a).

Corollary 2.11. Suppose $\chi$ is not of the determinant type, and $A \in M_{n}$ is invertible. Then $A$ is normal if and only if $K(A)$ is normal.

Proof. The necessity part is clear. To prove the converse, suppose $K(A)$ is normal. Then Theorem 2.10 (a) or (b) holds. If (b) holds, with $\operatorname{rank}(A)<n$, then $A$ is singular, contradicting the assumption on $A$. If (b) holds with $\operatorname{rank}(A)=n$, then $\chi$ is of the determinant type, contradicting the assumption on $\chi$. Hence, we see that (a) holds, and the result follows.

By Corollary 2.7 if every element $\alpha$ in $\bar{\Delta}$ satisfies $m_{1}(\alpha)=\cdots=m_{n}(\alpha)$, then $n=\mu(\bar{\Delta})$ and $K(A)=\operatorname{det}(A)^{m / n} I_{|\bar{\Delta}|}$ for all $A \in M_{n}$. Consequently, $K(A)$ is positive definite (respectively, unitary) if and only if $\operatorname{det}(A)^{m / n}>0$ (respectively, $|\operatorname{det}(A)|=1)$. Apart from this trivial case, we will show that $K(A)$ is a nonzero multiple of a positive definite (respectively, unitary) matrix if and only if $A$ is. To achieve this goal, we need the concept of majorization and a result from [10]. Given two real vectors $x, y \in \mathbb{R}^{n}$, we say that $x$ is majorized by $y$ if the sum of entries of the two vectors are the same, and the sum of the $k$ largest entries of $x$ is not larger than that of $y$ for $k=1, \ldots, n-1$. The following result follows from the Corollary in [10].

Lemma 2.12. Let $\alpha \in \bar{\Delta}$, and let $\left(m_{1}^{\prime}, \ldots, m_{n}^{\prime}\right)$ be a vector of positive integers majorized by $\left(m_{1}(\alpha), \ldots, m_{n}(\alpha)\right)$. Then there exists $\beta \in \bar{\Delta}$ such that $\left(m_{1}(\beta), \ldots\right.$, $\left.m_{n}(\beta)\right)=\left(m_{1}^{\prime}, \ldots, m_{n}^{\prime}\right)$.

We are now ready to prove the promised results.

Theorem 2.13. Suppose $\chi$ is not of the determinant type, and $A \in M_{n}$. Then there exists $\eta \in \mathbb{C}$ with $|\eta|=1$ such that $\eta K(A)$ is positive definite if and only if there exists $\xi \in \mathbb{C}$ with $\xi^{m}=\eta$ such that $\xi A$ is positive definite. 
Proof. The sufficiency part is clear. To prove the converse, suppose $\eta K(A)$ is positive definite, where $\eta \in \mathbb{C}$ with $|\eta|=1$. Then $K(A)$ is normal. Since $\chi$ is not of the determinant type and $K(A)$ is invertible, by Corollary 2.11 we see that $A$ is normal. Furthermore, if $A$ has eigenvalues $\lambda_{1}, \ldots, \lambda_{n}$, then none of them is zero.

To complete the proof, we show that there exists $\xi \in \mathbb{C}$ such that $\xi^{m}=\eta$ and $\lambda_{j}=\xi\left|\lambda_{j}\right|$ for all $1 \leq j \leq n$, as follows. Since $\chi$ is not of the determinant type, there exists $\alpha \in \bar{\Delta}$ such that $m_{p}(\alpha)<m_{q}(\alpha)$ for some $1 \leq p<q \leq n$. By Lemma 2.12, there exists $\beta \in \bar{\Delta}$ such that $\left(m_{p}(\beta), m_{q}(\beta)\right)=\left(m_{p}(\alpha)+1, m_{q}(\alpha)-1\right)$ and $m_{t}(\beta)=m_{t}(\alpha)$ for $t \neq p, q$. Now, for any $1<j \leq n$, there exists a permutation $\sigma \in S_{n}$ so that $\sigma(p)=1$ and $\sigma(q)=j$. Furthermore, by Proposition 1.4 (f), we see that $\lambda_{\alpha}=\prod_{i=1}^{n} \lambda_{\sigma(i)}^{m_{i}(\alpha)}$ and $\lambda_{\beta}=\prod_{i=1}^{n} \lambda_{\sigma(i)}^{m_{i}(\beta)}$ are eigenvalues of $K(A)$. It follows that $\eta \lambda_{\alpha}$ and $\eta \lambda_{\beta}$ are eigenvalues of $\eta K(A)$, and hence both of them are positive. Consequently,

$$
\lambda_{\alpha} / \lambda_{\beta}=\lambda_{j} / \lambda_{1}>0 .
$$

Thus, all the eigenvalues of $A$ have the same argument, i.e., $\xi A$ is positive definite for some $\xi \in \mathbb{C}$ with $|\xi|=1$. Since both $K(\xi A)=\xi^{m} K(A)$ and $\eta K(A)$ are positive definite, we see that $\xi^{m}=\eta$ as asserted.

Remark 2.14. In [55], the author attempted to prove the above theorem with $\eta=1$ under the assumption that $\mu(\bar{\Delta})<n$. Unfortunately, there is a gap in the proof. Note that in p. 203 of [55], the author tried to conclude that all the eigenvalues $\lambda_{1}, \ldots, \lambda_{n}$ of $A$ have the same argument based on the fact that there exists $\beta \in$ $\bar{\Delta} \cap \Gamma_{m, r}$, where $r=\mu(\bar{\Delta})<n$, satisfying

$$
\lambda_{i}^{m_{k}(\beta)}=\lambda_{k}^{m_{k}(\beta)}
$$

for all $k$ with nonzero $m_{k}(\beta)$ (cf. equation (8) in [55]). However, using our Example 2.9 (b), one can check that such a deduction is not valid. In fact, for $A=\operatorname{diag}(1,1,-1)$, since the only $\beta$ in $\bar{\Delta} \cap \Gamma_{m, r}$ is $(1,1,2,2)$, we see that (2) is satisfied; but clearly the eigenvalues of $A$ do not have the same argument. One actually has to use the deeper result in [10] to get the desired conclusion as in our proof.

Theorem 2.15. Suppose $\chi$ is not of the determinant type. Let $A \in M_{n}$. Then $K(A)$ is unitary or a scalar matrix if and only if $A$ has the corresponding property.

Proof. The sufficiency part is clear. To prove the converse, suppose $K(A)$ is unitary (respectively, a scalar matrix). Since $\chi$ is not of the determinant type and $K(A)$ is normal, by Corollary 2.11 we see that $A$ is normal. Suppose $A$ has eigenvalues $\lambda_{1}, \ldots, \lambda_{n}$. One can use arguments similar to those in the proof of Theorem 2.13 to show that $\left|\lambda_{j} / \lambda_{1}\right|=1$ (respectively, $\lambda_{j} / \lambda_{1}=1$ ) for all $j=2, \ldots, n$. The result follows.

Theorem 2.16. Let $r=\mu(\bar{\Delta})$ and $A \in M_{n}$ with $\operatorname{rank}(A) \geq r$. Then there exists $\eta \in \mathbb{C}$ with $|\eta|=1$ such that $\eta K(A)$ is (i) hermitian, (ii) positive semi-definite, or (iii) an orthogonal projection if and only if one of the following holds:

(a) There exists $\xi \in \mathbb{C}$ with $\xi^{m}=\eta$ such that $\xi A$ has the corresponding property.

(b) $\chi$ is of the special type, and $A$ is unitarily similar to $A_{1} \oplus 0_{n-r}$ such that $\eta \operatorname{det}\left(A_{1}\right)^{m / r}$ is (i) real, (ii) nonnegative, or (iii) is equal to 1 . 
Proof. The sufficiency part is clear. Conversely, if $K(A)$ satisfies (i), (ii), or (iii), then $K(A)$ is normal. Hence $A$ satisfies condition (a) or (b) of Theorem [2.10. If Theorem 2.10 (a) holds, then $A$ is normal. One can use arguments similar to those in the proof of Theorem 2.13 to show that condition (a) of this theorem holds. If Theorem 2.10 (b) holds, one easily checks that condition (b) of Theorem 2.16 holds.

Note that Theorem 2.16 (i) covers the special cases when $K(A)$ is hermitian or skew-hermitian. Next, we determine the conditions for two induced matrices to be equal.

Theorem 2.17. Let $r=\mu(\bar{\Delta})$. Then $A, B \in M_{n}$ satisfy $K(A)=K(B)$ if and only if one of the following holds:

(a) $\operatorname{rank}(A)<r$ and $\operatorname{rank}(B)<r$.

(b) There exists $\xi \in \mathbb{C}$ with $\xi^{m}=1$ such that $B=\xi A$.

(c) $\chi$ is of the special type, and there are unitary matrices $U, V \in M_{n}$ such that $U A V=A_{1} \oplus 0_{n-r}$ and $U B V=B_{1} \oplus 0_{n-r}$ with $\operatorname{det}\left(A_{1}\right)^{m / r}=\operatorname{det}\left(B_{1}\right)^{m / r}$.

Proof. If (a) or (b) holds, then clearly $K(A)=K(B)$. If (c) holds, then

$$
K(U) K(A) K(V)=K(U) K(B) K(V)
$$

and hence $K(A)=K(B)$. Conversely, suppose $K(A)=K(B)$. If $K(A)=$ $K(B)=0$, then (a) holds. Otherwise, let $U, V \in M_{n}$ be unitary such that $U A V=\operatorname{diag}\left(a_{1}, \ldots, a_{k}, 0, \ldots, 0\right)$, where $k \geq r$ and $a_{1} \geq \cdots \geq a_{k}>0$. Suppose $D=\operatorname{diag}\left(1 / a_{1}, \ldots, 1 / a_{k}, 1, \ldots, 1\right)$. Then

$$
\begin{aligned}
K\left(I_{k} \oplus 0_{n-k}\right) & =K(U A V D) \\
& =K(U) K(A) K(V) K(D) \\
& =K(U) K(B) K(V) K(D) \\
& =K(U B V D)
\end{aligned}
$$

is an orthogonal projection. Then Theorem 2.16 (a) or (b.iii) holds.

If Theorem 2.16 (b.iii) holds, then $k=r$ and $U B V D$ is unitarily similar to $C \oplus 0_{n-r}$ such that $\operatorname{det}(C)^{m / r}=1$. Suppose

$$
U B V D=\left(\begin{array}{ll}
C_{1} & C_{2} \\
C_{3} & C_{4}
\end{array}\right)
$$

with $C_{1} \in M_{r}$. Comparing the $(\alpha, \alpha)$ entry for some $\alpha \in \bar{\Delta} \cap \Gamma_{m, r}$ on the left and right side of the matrices

$$
K\left(I_{k} \oplus 0_{n-k}\right)=K(U B V D),
$$

and using the fact that $\chi$ is of the special type, we see that

$$
1=\left(\zeta_{1} \cdots \zeta_{r}\right)^{m / r}=\operatorname{det}\left(C_{1}\right)^{m / r},
$$

where $\zeta_{1}, \ldots, \zeta_{r}$ are the eigenvalues of $C_{1}$. Thus $\left|\operatorname{det}\left(C_{1}\right)\right|=|\operatorname{det}(C)|$ is the product of the $r$ largest singular values of $C \oplus 0_{n-r}$, which is unitarily similar to $U B V D$. By [28, Theorem 4], we see that $U B V D=C_{1} \oplus C_{4}$. Since $U B V D$ is unitarily similar to $C \oplus 0_{n-r}$ and has rank $r$, we see that $C_{4}=0_{n-r}$. Thus condition (c) of the theorem holds with $A_{1}=\operatorname{diag}\left(a_{1}, \ldots, a_{r}\right)$ and $B_{1}=C_{1} A_{1}$. 
If Theorem [2.16 (a) holds, then $\zeta U B V D$ is an orthogonal projection for some $\zeta \in \mathbb{C}$ with $\zeta^{m}=1$. Suppose

$$
\zeta U B V D=\left(\begin{array}{ll}
C_{1} & C_{2} \\
C_{2}^{*} & C_{3}
\end{array}\right)
$$

with $C_{1} \in M_{k}$. We claim that $C_{1}=I_{k}$, and hence $\zeta U B V D=I_{k} \oplus 0_{n-k}$. If our claim is not true, then $C_{1}$ has eigenvalues $c_{1} \geq \cdots \geq c_{k}$ with $1 \geq c_{1}$ and $1>c_{k} \geq 0$. By Theorem 2.16] and Proposition 1.4 (h), the matrix $K\left(C_{1} \oplus 0\right)$ cannot be an orthogonal projection of rank $\left|\bar{\Delta} \cap \Gamma_{m, k}\right|$. But then

$$
\begin{aligned}
K\left(I_{k} \oplus 0_{n-k}\right) & =\left[K\left(I_{k} \oplus 0_{n-k}\right)\right]^{3} \\
& =K\left(I_{k} \oplus 0_{n-k}\right) K(\zeta U B V D) K\left(I_{k} \oplus 0_{n-k}\right) \\
& =K\left[\left(I_{k} \oplus 0_{n-k}\right)(\zeta U B V D)\left(I_{k} \oplus 0_{n-k}\right)\right] \\
& =K\left(C_{1} \oplus 0_{n-k}\right),
\end{aligned}
$$

which is a contradiction. Thus our claim is proved and condition (b) of the theorem holds with $\xi=\bar{\zeta}$.

The results in this section explain why if $\chi$ is the principal character or if $\operatorname{rank}(A)>m$, then (I)-(III) hold. Also, one sees why (I)-(III) fail if $\chi$ is the alternate character on $S_{m}$. In particular, we have the following corollary.

Corollary 2.18. Suppose $\chi$ is not of the determinant type.

(a) Let $A, B \in M_{n}$ be such that

$$
\operatorname{rank}(A) \geq \begin{cases}\mu(\bar{\Delta})+1 & \text { if } \chi \text { is of the special type, } \\ \mu(\bar{\Delta}) & \text { otherwise. }\end{cases}
$$

Then (I)-(III) hold.

(b) If $\chi$ is of the special type, then there exist $A, B \in M_{n}$ (with ranks equal to $\mu(\bar{\Delta}))$ such that all of (I)-(III) fail.

\section{Decomposable Numerical Ranges and Decomposable Numerical Radi}

Let $A \in M_{n}$. The numerical range of $A$ is defined by

$$
W(A)=\left\{(A x, x): x \in \mathbb{C}^{n},(x, x)=1\right\},
$$

and the numerical radius of $A$ is defined by

$$
r(A)=\max \{|\eta|: \eta \in W(A)\} .
$$

These concepts have been studied extensively because of their connections and applications to many branches of pure and applied mathematics (see e.g., [19, 20. $23,24)$.

In the study of induced operators on symmetry tensors, it is natural to consider the decomposable numerical range of $A \in M_{n}$ defined by

$$
W_{\chi}(A)=\left\{\left(K(A) x^{*}, x^{*}\right): x^{*} \text { is a decomposable unit tensor }\right\},
$$

(see [37, 43]) and the decomposable numerical radius of $A \in M_{n}$ defined by

$$
r_{\chi}(A)=\max \left\{|\eta|: \eta \in W_{\chi}(A)\right\} .
$$


In terms of the generalized matrix function, we can write

$$
W_{\chi}(A)=\left\{\frac{d_{\chi}\left(X^{*} A X\right)}{d_{\chi}\left(X^{*} X\right)}: X \in M_{n \times m}, d_{\chi}\left(X^{*} X\right) \neq 0\right\} .
$$

Evidently, when $m=1, W_{\chi}(A)$ reduces to the classical numerical range $W(A)$.

Certainly, one can also consider the classical numerical range and radius of the induced matrix $K(A)$. Since the set of decomposable unit tensors is usually a proper subset of the set of unit vectors in $V_{\chi}^{m}(H)$, we have

$$
W_{\chi}(A) \subseteq W(K(A)),
$$

and the inclusion is often strict. Consequently, we have

$$
r_{\chi}(A) \leq r(K(A)),
$$

and again the inequality is usually strict. Thus, the set $W_{\chi}(A)$ usually contains "less" information than $W(K(A))$, and the quantity $r_{\chi}(A)$ is different from $r(K(A))$. However, in the study of symmetry classes of tensors and induced operators, one may only have information about $W_{\chi}(A)$ and $r_{\chi}(A)$ but not $W(K(A))$ and $r(K(A))$. It is natural to ask if we can still extract information about the operator $A$ based on the limited knowledge on $W_{\chi}(A)$ and $r_{\chi}(A)$. This chapter is devoted to the study of this line of questions. In particular, we show that many results on the classical numerical range and radius can actually be extended to decomposable numerical ranges and radii.

3.1. Decomposable Numerical Ranges with Special Geometrical Features. The numerical range is a useful tool for studying matrices and operators. In particular, there is an interesting interplay between the geometric properties of $W(A)$ and the algebraic properties of a matrix $A$. For example, we have the following result (see e.g. [23]).

Proposition 3.1. Let $A \in M_{n}$.

(a) $W(A)=\{\lambda\}$ if and only if $A=\lambda I$.

(b) $W(A) \subseteq \mathbb{R}$ if and only if $A$ is hermitian.

(c) $W(A) \subseteq(0, \infty)$ if and only if $A$ is positive definite.

(d) $W(A)$ has no interior point if and only if $A$ is a normal matrix with eigenvalues lying on a straight line.

There has been attempts to extend this result to decomposable numerical ranges (see [5, 54, 55]). We list the results on the decomposable numerical ranges associated with the alternate character $\varepsilon$ on $S_{m}$ (see [54] and [5, §4]) that will be useful in our subsequent discussion. To avoid trivial consideration, we assume that $m<n$; otherwise, we have $W_{\varepsilon}(A)=\{\operatorname{det}(A)\}$.

Proposition 3.2. Suppose $1<m<n$ and $\varepsilon$ is the alternate character on $H=S_{m}$. Let $A \in M_{n}$. Then $W_{\varepsilon}(A)=\{\eta\}$ if and only if one of the following conditions holds.

(a) $\eta=0$ and $A$ has rank less than $m$.

(b) $A=\lambda I$ so that $\lambda^{m}=\eta$.

Proposition 3.3. Suppose $1<m<n$ and $\varepsilon$ is the alternate character on $H=$ $S_{m}$. Let $A \in M_{n}$. Then $W_{\varepsilon}(A)$ is a non-degenerate line segment if and only if $\operatorname{rank}(A) \geq m$ and one of the following conditions holds.

(a) $A$ is unitarily similar to $A_{1} \oplus 0_{n-m}$ so that $\operatorname{det}\left(A_{1}\right) \neq 0$.

(b) $\eta A$ is hermitian for some nonzero $\eta \in \mathbb{C}$. 
(c) $1<m<n-1$ and $A$ is unitarily similar to $a I_{n-1} \oplus[b]$ so that $a, b \in \mathbb{C}$ do not lie on a line passing through the origin.

(d) $1<m=n-1$ and $A$ is invertible so that the eigenvalues of $A^{-1}$ lie on a straight line not passing through the origin.

To generalize Proposition 3.1 to other types of decomposable numerical ranges, we need the result of Robinson [50] (see also [56]).

Proposition 3.4. A linear operator $L$ on $V_{\chi}^{m}(H)$ satisfies $\left(L v^{*}, v^{*}\right)=0$ for all decomposable tensors $v^{*}=v_{1} * \cdots * v_{n} \in V_{\chi}^{m}(H)$ if and only if $L=0$. As a result, the following conditions are equivalent for a matrix $A \in M_{n}$.

(a) $W_{\chi}(A)=\{0\}$.

(b) $r_{\chi}(A)=0$.

(c) $K(A)=0$, i.e., $\operatorname{rank}(A)<\mu(\bar{\Delta})$.

Now, we are ready to prove the main results of this section. Note that the results are also valid if we replace $W_{\chi}(A)$ by $W(K(A))$ using similar (simpler) proofs.

Theorem 3.5. Let $r=\mu(\bar{\Delta})$ and $A \in M_{n}$ with $\operatorname{rank}(A) \geq r$. Then there exists $\eta \in \mathbb{C}$ with $|\eta|=1$ such that $W_{\chi}(A)$ is a subset of (i) $\eta \mathbb{R}$ or (ii) $\eta[0, \infty$ ) if and only if one of the following conditions holds.

(a) There exists $\xi \in \mathbb{C}$ with $\xi^{m}=\bar{\eta}$ such that $\xi A$ is (i) hermitian or (ii) positive semi-definite.

(b) $\chi$ is of the special type and $A$ is unitarily similar to $A_{1} \oplus 0_{n-r}$ such that $\bar{\eta} \operatorname{det}\left(A_{1}\right)^{m / r}$ is (i) real or (ii) nonnegative.

Proof. If $\chi$ and $A$ satisfy (a) or (b), then $W_{\chi}(A)$ is a subset of $\eta \mathbb{R}$ or $\eta[0, \infty)$. Conversely, suppose $W_{\chi}(A) \subseteq \eta \mathbb{R}$ or $\eta[0, \infty)$. Then

$$
\left(\left(\eta K(A)^{*}-\bar{\eta} K(A)\right) v^{*}, v^{*}\right)=0
$$

for all unit decomposable tensors $v^{*}$. By Proposition 3.4 $\bar{\eta} K(A)-\eta K(A)^{*}=0$, i.e., $\bar{\eta} K(A)$ is hermitian. Applying Theorem 2.16, we see that $A$ satisfies (a) or (b).

By Theorem 3.5, we have the following corollary.

Corollary 3.6. Let $A \in M_{n}$. Then $A$ is (i) hermitian, (ii) positive definite, (iii) positive semi-definite if and only if $W_{\chi}(A+\eta I) \subseteq S$ for all $\eta \in S$ with

$$
\text { (i) } S=\mathbb{R} \text {, (ii) } S=(0, \infty) \text {, (iii) } S=[0, \infty) \text {. }
$$

Similarly, one can use Theorem 2.13 to prove the following.

Theorem 3.7. Suppose $\chi$ is not of the determinant type. Let $A \in M_{n}$.

(a) $W_{\chi}(A) \subseteq \eta(0, \infty)$ if and only if there exists $\xi \in \mathbb{C}$ with $\xi^{m}=\bar{\eta}$ such that $\xi A$ is positive definite.

(b) $W_{\chi}(A)$ is a singleton if and only if $A$ is a scalar matrix.

By Theorem [3.5 we know that $W_{\chi}(A)$ is a subset of a line passing through the origin if and only if $\xi A$ is hermitian for some $\xi \in \mathbb{C}$. Next, we determine the condition under which $W_{\chi}(A)$ is a subset of a line not passing through the origin. The result covers Proposition 3.3 (c) and (d). 
Theorem 3.8. Suppose $\chi$ is not of the determinant type, and $A \in M_{n}$ is nonscalar. Then $W_{\chi}(A)$ is a subset of a line not passing through the origin if and only if for every $\alpha \in \bar{\Delta}$,

$$
\left(m_{1}(\alpha), \ldots, m_{n}(\alpha)\right) \text { is a permutation of }(k, \ldots, k, \underbrace{k+1, \ldots, k+1}_{t}),
$$

where $m=n k+t$ with $1 \leq t<n$, and $A$ is an invertible normal matrix such that one of the following holds:

(a) $t=1$ and the eigenvalues of $A$ lie on a line not passing through the origin;

(b) $1<t<n-1$ and one of the eigenvalues of $A$ has multiplicity $n-1$;

(c) $t=n-1$ and the eigenvalues of $A^{-1}$ lie on a line not passing through the origin.

Proof. Suppose every $\alpha \in \bar{\Delta}$ satisfies (3), and $A$ is an invertible normal matrix with eigenvalues $\lambda_{1}, \ldots, \lambda_{n}$. Then $K(A)$ has eigenvalues

$$
\operatorname{det}(A)^{k} \lambda_{i_{1}} \ldots \lambda_{i_{t}}, \quad \text { with } 1 \leq i_{1}<\cdots<i_{t} \leq n .
$$

If (a), (b) or (c) holds, one easily checks that (cf. Proposition 3.3) the eigenvalues of $K(A)$ lie on a line not passing through the origin. Since $W_{\chi}(A) \subseteq W(K(A))$ and $W(K(A))$ is the convex hull of the eigenvalues of $K(A)$, we see that $W_{\chi}(A)$ is a subset of a line not passing through the origin.

Conversely, suppose $W_{\chi}(A)$ is a subset of a line not passing through the origin. Then there exists $c, d \in \mathbb{C}$ with $|c|=1$ such that for any $z \in W_{\chi}(A)$, we have $c z+d \in \mathbb{R}$, i.e.,

$$
\begin{aligned}
0 & =(c z+d)-(c z+d)^{*} \\
& =\left(\left(c K(A)+d I-\bar{c} K(A)^{*}-\bar{d} I\right) x^{*}, x^{*}\right)
\end{aligned}
$$

for all unit decomposable tensors $x^{*}$. By Proposition $3.4 c K(A)+d I$ is hermitian, and thus $K(A)$ is normal. Thus, Theorem 2.10 (a) or (b) holds. Note that $0 \notin$ $W_{\chi}(A)$. Since $W_{\chi}(A)$ contains all the eigenvalues of $K(A)$ (see e.g. [55, pp. 200201]), we see that $K(A)$ is invertible. By Corollary 2.11 and the fact that $\chi$ is not of the determinant type, we see that $A$ is an invertible normal matrix.

Suppose $A$ has (nonzero) eigenvalues $\lambda_{1}, \ldots, \lambda_{n}$. If (3) is not satisfied, then there exists $\alpha \in \bar{\Delta}$ such that $m_{p}(\alpha)-m_{q}(\alpha)=s>1$ for some $1 \leq p<q \leq n$. Then by Lemma 2.12 there exists $\beta \in \bar{\Delta}$ with $\left(m_{p}(\beta), m_{q}(\beta)\right)=\left(m_{p}(\alpha)-1, m_{q}(\alpha)+1\right)$ and $m_{j}(\alpha)=m_{j}(\beta)$ for other $j$. Now, for any $\lambda_{i} \neq \lambda_{1}$, there exists a permutation $\sigma \in S_{n}$ so that $\sigma(p)=1$ and $\sigma(q)=i$. Let $\tilde{\sigma}$ be obtained from $\sigma$ by interchanging the image of $\sigma(p)$ and $\sigma(q)$. Then

$$
\eta=\prod_{j=1}^{n} \lambda_{\sigma(j)}^{m_{j}(\alpha)}, \quad \eta\left(\lambda_{i} / \lambda_{1}\right)^{s}=\prod_{j=1}^{n} \lambda_{\tilde{\sigma}(j)}^{m_{j}(\alpha)} \quad \text { and } \quad \eta\left(\lambda_{i} / \lambda_{1}\right)=\prod_{j=1}^{n} \lambda_{\sigma(j)}^{m_{j}(\beta)}
$$

are eigenvalues of $K(A)$ and hence belong to $W_{\chi}(A)$. Thus, they are collinear.

Let $z=\lambda_{i} / \lambda_{1}$. By the discussion in the previous paragraph, we see that

$$
1, z, z^{s}
$$

are collinear. If $s>2$, then by Lemma 2.12 there exists $\gamma \in \bar{\Delta}$ such that $\left(m_{p}(\gamma), m_{q}(\gamma)\right)=\left(m_{p}(\alpha)-2, m_{q}(\alpha)+2\right)$ and $m_{j}(\alpha)=m_{j}(\gamma)$ for other $j$. Repeating the previous argument, we see that

$$
1, z, z^{2}, z^{s}
$$


are collinear. Thus, $z^{2}-1$ is a real multiple of $z-1$, i.e., $z+1$ is real. Hence $z=\lambda_{i} / \lambda_{1}$ is real as well. Since this is true for all $i \leq n$ with $\lambda_{i} \neq \lambda_{1}$, we see that $\lambda_{1}^{-1} A$ is a hermitian matrix, and $W_{\chi}(A)$ will lie on a line passing through the origin, which is a contradiction.

Now, we see that every $\alpha \in \bar{\Delta}$ satisfies (3). It follows that $K(A)$ has eigenvalues

$$
\operatorname{det}(A)^{k} \lambda_{i_{1}} \ldots \lambda_{i_{t}}, \quad \text { with } 1 \leq i_{1}<\cdots<i_{t} \leq n
$$

and they are lying on a line not passing through the origin. This reduces to the analysis of the conditions under which the $n$ nonzero complex numbers $\lambda_{1}, \ldots, \lambda_{n}$ will generate numbers

$$
\lambda_{i_{1}} \ldots \lambda_{i_{t}}, \quad \text { with } 1 \leq i_{1}<\cdots<i_{t} \leq n,
$$

that are collinear. Evidently, this is exactly the same condition for the decomposable numerical range of $A$ associated with the alternate character on $S_{t}$ to be on a straight line not passing through the origin. By Proposition [3.3, we get the conclusion.

3.2. Matrix Inequalities and Equalities. Multilinear techniques are very useful in deriving matrix inequalities (see e.g. [37, Section 8.3] and the reference therein). Many obscure matrix inequalities become clear once they are put in the multilinear algebraic settings. In fact, there are many interesting results and questions along this line of study. In this section, we focus on those inequalities related to the spectral radius $\rho(K(A))$, spectral norm $\|K(A)\|$, the numerical radius $r(K(A))$, and the decomposable numerical radius $r_{\chi}(A)$.

The following result is well known for the classical case (see e.g. [23]).

Proposition 3.9. Let $A \in M_{n}$. Then

$$
\rho(A) \leq r(A) \leq\|A\| \text {. }
$$

(a) The equality $r(A)=\|A\|$ holds if and only if $\rho(A)=\|A\|$. This happens if and only if $A$ is unitarily similar to $r(A) V \oplus B$ for some unitary $V \in M_{k}$ and some $B \in M_{n-k}$ satisfying $\|B\|<r(A)$.

(b) The equality $\rho(A)=r(A)$ holds if and only if $A$ is unitarily similar to $r(A) V \oplus$ $B$ for some unitary $V \in M_{k}$ and some $B \in M_{n-k}$ satisfying $r(B)<r(A)$.

We say that $A \in M_{n}$ is spectral if $\rho(A)=r(A)$, and $A \in M_{n}$ is radial if $\rho(A)=$ $\|A\|$. These classes of matrices have attracted the attention of many researchers (see e.g. [20, 23, 24] and their references). At one point, researchers also gave a name to those matrices satisfying $\|A\|=r(A)$. Later, Wintner [58] showed that such matrices are just radial matrices (cf. Proposition 3.9 (a)). There has been a great deal of interest in extending Proposition 3.9 and the concepts of spectral and radial matrices in the contexts of symmetry classes of tensors; see [1, 4, 28, 38, 50, 52. and the references therein. We summarize the result in 52 in the next proposition. It is worth noting that the result of Wintner has a nice extension (cf. Proposition $3.10(\mathrm{~b}))$.

Proposition 3.10. Let $A \in M_{n}$. Then

$$
\rho(K(A)) \leq r_{\chi}(A) \leq r(K(A)) \leq\|K(A)\| .
$$

Suppose $\operatorname{rank}(A) \geq r=\mu(\bar{\Delta})$.

(a) The value $\rho(K(A))$ equals

$$
g(A)=r_{\chi}(A) \quad \text { or } \quad r(K(A))
$$


if and only if $A$ is unitarily similar to $A_{1} \oplus A_{2}$ such that $A_{1} \in M_{r}$ has eigenvalues $\lambda_{1}, \ldots, \lambda_{r}$ and $g(A)=\left|\prod_{j=1}^{r} \lambda_{j}^{m_{j}(\alpha)}\right|$.

(b) The value $\|K(A)\|$ equals any (and hence all) of

$$
\rho(K(A)), \quad r_{\chi}(A) \quad \text { or } \quad r(K(A))
$$

if and only if $A$ is unitarily similar to $A_{1} \oplus A_{2}$ such that $A_{1} \in M_{r}$ has eigenvalues $\lambda_{1}, \ldots, \lambda_{r}$ and $\|K(A)\|=\left|\prod_{j=1}^{r} \lambda_{j}^{m_{j}(\alpha)}\right|$ for some $\alpha \in \bar{\Delta}$.

In the following we add one more term, namely, $|\operatorname{det}(A)|^{m / n}$ to the chain of inequalities (4), and study the equality cases. The extreme cases give rise to some new characterizations of multiples of unitary matrices, which are useful in the next chapter. To avoid trivial consideration, we assume that $\chi$ is not of the determinant type and $K(A) \neq 0$.

Theorem 3.11. Suppose $\chi$ is not of the determinant type, and $\operatorname{rank}(A) \geq r=$ $\mu(\bar{\Delta})$. Then

$$
|\operatorname{det}(A)|^{m / n} \leq \rho(K(A)) \leq r_{\chi}(A) \leq r(K(A)) \leq\|K(A)\| .
$$

(a) The equality $|\operatorname{det}(A)|^{m / n}=\rho(K(A))$ holds if and only if all eigenvalues of $A$ have the same magnitude.

(b) The value $|\operatorname{det}(A)|^{m / n}$ is equal to any (and hence all) of

$$
r_{\chi}(A), \quad r(K(A)) \quad \text { or } \quad\|K(A)\|
$$

if and only if $\eta A$ is a unitary matrix for some nonzero $\eta \in \mathbb{C}$.

Proof. The inequalities and part (a) is clear. We focus on the proof of (b). The sufficiency part is clear. To prove the converse, suppose $|\operatorname{det}(A)|^{m / n}$ is equal to $r_{\chi}(A), r(K(A))$ or $\|K(A)\|$. Then $|\operatorname{det}(A)|^{m / n}=r_{\chi}(A)>0$ by Proposition 3.4 We may assume that $|\operatorname{det}(A)|=1$; otherwise, replace $A$ by $\gamma A$ for some suitable $\gamma>0$. Then we have

$$
1=|\operatorname{det}(A)|=r_{\chi}(A) .
$$

To prove that $A$ is unitary, we show that all the singular values of $A$ equal 1 . Suppose it is not true. Then $A$ has singular values $s_{1} \geq \cdots \geq s_{n}$ such that $s_{1}>s_{n}$. Let $A$ have polar decomposition $P U$ so that $U$ is unitary and $P$ is positive semidefinite with eigenvalues $s_{1}, \ldots, s_{n}$. Then there exists a unitary matrix $V$ such that $V^{*} U V=\operatorname{diag}\left(\eta_{1}, \ldots, \eta_{n}\right)$ with $\left|\eta_{j}\right|=1$ for all $j=1, \ldots, n$. Since

$$
K\left(V^{*} A V\right)=K\left(V^{*} P V\right) K\left(V^{*} U V\right),
$$

the diagonal entries of $K\left(V^{*} A V\right)$ are of the form $d_{1} \eta_{1}, \ldots, d_{s} \eta_{s}$, with $s=|\bar{\Delta}|$, where $d_{1}, \ldots, d_{s}$ are diagonal entries of $K\left(V^{*} P V\right)$ and $K\left(V^{*} U V\right)=\operatorname{diag}\left(\eta_{1}, \ldots, \eta_{s}\right)$. Note that all the diagonal entries of $K\left(V^{*} A V\right)$ belong to $W_{\chi}(A)$ and the sum of their moduli equals

$$
\sum_{j=1}^{s}\left|d_{j} \eta_{j}\right|=\sum_{j=1}^{s} d_{j}=\operatorname{tr} K\left(V^{*} P V\right)=\sum_{j=1}^{s} \lambda_{j}(K(P)),
$$

where $\lambda_{1}(K(P)) \geq \cdots \geq \lambda_{s}(K(P))$ are the eigenvalues of $K(P)$. By Corollary 2.7 there exists $\beta \in \bar{\Delta}$ such that $m_{1}(\beta)>m_{n}(\beta)$. By the fact that $s_{1}>s_{n}$, we see that 
$\prod_{j=1}^{n} s_{j}^{m_{j}(\beta)}$ and $s_{1}^{m_{n}(\beta)} s_{n}^{m_{1}(\beta)} \prod_{j=2}^{n-1} s_{j}^{m_{j}(\beta)}$ are eigenvalues of $K(P)$ with different magnitudes. Thus, not all $\lambda_{j}(K(P))$ 's are equal. Since

$$
\prod_{j=1}^{s} \lambda_{j}(K(P))=|\operatorname{det}(K(P))|=|\operatorname{det}(K(A))|=|\operatorname{det}(A)|^{k}=1,
$$

where $k=|\bar{\Delta}| m / n$, we conclude that $\sum_{j=1}^{s} \lambda_{j}(K(P))>s$. As a result, one of the diagonal entries of $K\left(V^{*} A V\right)$ has magnitude larger than 1, and hence $r_{\chi}(A)>$ 1.

In [40, Theorem 1], it was shown that when $m<n$ and $\chi$ is the alternate character, a matrix $A \in M_{n}$ is unitary if and only if all the eigenvalues of $A$ have magnitude one and $r_{\chi}(A) \leq 1$. By the above result, we have the following corollary.

Corollary 3.12. Suppose $\chi$ is not of the determinant type. Let $A \in M_{n}$ be such that all eigenvalues of $A$ or $K(A)$ have the same magnitude not equal to zero. Then the following conditions are equivalent.

(a) $A$ is a (nonzero) multiple of a unitary matrix.

(b) $A$ is normal.

(c) $0 \neq\|K(A)\|$ equals $r_{\chi}(A)$ or $r(K(A))$.

(d) $0 \neq \rho(K(A))$ equals any one (and hence all) of the following:

$$
r_{\chi}(A), \quad r(K(A)), \quad \text { or } \quad\|K(A)\| .
$$

Proof. The implication (a) $\Longrightarrow(\mathrm{b})$ is clear. Suppose (b) holds. Then $\|K(A)\|=$ $\rho(K(A))$. By Proposition 3.10 (b), condition (c) follows.

Suppose (c) holds. Then $\|K(A)\|=r(K(A))$. Applying Theorem 3.9 to $K(A)$, we see that $\|K(A)\|=\rho(K(A))$. By (4), condition (d) holds.

Suppose (d) holds. Then $\rho(K(A))=r_{\chi}(A)$. Since all the eigenvalues of $K(A)$ have the same magnitude and $\operatorname{det}(K(A))$ is the product of the eigenvalues of $K(A)$, we have $\rho(K(A))=|\operatorname{det}(K(A))|^{1 /|\bar{\Delta}|}=|\operatorname{det}(A)|^{m / n}$. Thus, we have $r_{\chi}(A)=$ $\rho(K(A))=|\operatorname{det}(A)|^{m / n}$. By Theorem 3.11(b), we get condition (a).

Next, we extend some results in 39 and [52 concerning the characterization of unitary matrices $A$ in terms of equalities $1=r_{\chi}(A)=r_{\chi}(U A V)$ for all unitary $U, V \in M_{n}$. In [39], the result was obtained for the case when $m<n$ and $\chi$ is the alternate character. In [52, the result was extended to those $\chi$ for which there exists $r<n$ such that $\Gamma_{m, r} \cap \bar{\Delta} \neq \emptyset$. We relax all these conditions in our result.

Corollary 3.13. Suppose $\chi$ is not of the determinant type. Let $A \in M_{n}$ be such that $K(A) \neq 0$. Then $A$ is a (nonzero) multiple of a unitary matrix if and only if $0 \neq r_{\chi}(A)=r_{\chi}(U A V)$ for any unitary $U, V \in M_{n}$.

Proof. The necessity part is clear. Conversely, suppose $0 \neq r_{\chi}(A)=r_{\chi}(U A V)$ for any unitary $U, V \in M_{n}$. If $A$ is not a multiple of a unitary matrix, then $A$ has singular values $s_{1} \geq \cdots \geq s_{n}$ such that $s_{1}>s_{n}$. Let $A_{1}=U_{1} A V_{1}=\operatorname{diag}\left(s_{1}, \ldots, s_{n}\right)$ and $A_{2}=U_{2} A V_{2}=\sum_{j=1}^{n-1} s_{j} E_{j, j+1}+s_{n} E_{n, 1}$. Since $A_{1}$ is normal, by Proposition 3.10 (b) we have $\left\|K\left(A_{1}\right)\right\|=r_{\chi}\left(A_{1}\right)$. Note that all eigenvalues of $A_{2}$ have the same magnitude, and $A_{2}$ is not normal. By Corollary 3.12, we have

$$
r_{\chi}\left(A_{2}\right)<\left\|K\left(A_{2}\right)\right\|=\left\|K\left(A_{1}\right)\right\|=r_{\chi}\left(A_{1}\right)=r_{\chi}(A)=r_{\chi}\left(A_{2}\right),
$$

which is a contradiction. 


\section{Linear Preserver Problems}

An active area in matrix and operator theory is the linear preserver problems concerning the characterizations of linear operators with some special properties. For example, Frobenius [14] proved that a linear operator $L$ on $M_{n}$ satisfies

$$
\operatorname{det}(L(A))=\operatorname{det}(A) \quad \text { for all } A \in M_{n}
$$

if and only if there exist $M, N \in M_{n}$ with $\operatorname{det}(M N)=1$ so that $L$ is of the form

$$
A \mapsto M A N \quad \text { or } \quad A \mapsto M A^{t} N .
$$

The sufficiency part of the statement is clear. It is somewhat surprising that the two obvious transformations in (5) are the only admissible transformations on $M_{n}$ to $M_{n}$ preserving the determinant function.

Denote by $\operatorname{Eig}(A)$ the multiset (with $n$ elements) of eigenvalues of $A \in M_{n}$. In 41], the authors showed that a linear operator $L$ on $M_{n}$ satisfies

$$
\operatorname{Eig}(L(A))=\operatorname{Eig}(A) \quad \text { for all } A \in M_{n}
$$

if and only if there exists an invertible $S \in M_{n}$ so that $L$ is of the form

$$
A \mapsto S^{-1} A S \quad \text { or } \quad A \mapsto S^{-1} A^{t} S .
$$

Again, the sufficiency part of the statement is clear, and the interesting part is the converse. Furthermore, this result shows that the linear preservers of Eig(A) are actually Jordan isomorphisms on $M_{n}$, i.e., algebraic homomorphisms $L$ on $M_{n}$ satisfying $L\left(A^{2}\right)=L(A)^{2}$ for all $A \in M_{n}$.

Let us describe some more results related to our subsequent discussion. Specializing the results [27] and [46], we have the following equivalent conditions for a linear operator $L$ on $M_{n}$ :

(a) $L(I)=I$ and $\|L(A)\|=\|A\|$ for all $A \in M_{n}$.

(b) $W(L(A))=W(A)$ for all $A \in M_{n}$.

(c) There exists unitary $U \in M_{n}$ such that $L$ is of the form

$$
A \mapsto U^{*} A U \quad \text { or } \quad A \mapsto U^{*} A^{t} U .
$$

It is worth noting that in these cases, the linear preservers are actually Jordan homomorphisms on the $C^{*}$-algebra $M_{n}$.

To a certain extent, the above examples manifest the spirit of linear preserver problems, namely, one can often prove that the obvious linear maps are the only admissible linear preservers for a certain property or function, and the structure of linear preservers are often very elegant. Of course, there are situations that the linear preservers are not so well behaved. In such cases, it would be interesting to enumerate all the special cases and explain the pathological behaviors. We refer readers to the survey [47] on linear preserver problems.

In this chapter, we determine the structures of linear operators $L$ on $M_{n}$ such that

$$
F(L(A))=F(A) \quad \text { for all } A \in M_{n}
$$

where

$F(A)=\|K(A)\|, \rho(K(A)), r(K(A)), r_{\chi}(A), W(K(A)), W_{\chi}(A)$,

$\operatorname{Eig}(K(A))$ or $S p(K(A))$ - the spectrum of $K(A)$.

In all cases, we show that the linear preservers indeed behave well. A wide range of techniques are used in our proofs. Here, we mention some references for some existing results: 
(1) for linear preservers of $\|A\|$, see [27, 15, 34;

(2) for linear preservers of $\rho(A)$, see [8, 32];

(3) for linear preservers of $W_{\chi}(A)$ for special $\chi$, see [46, 40, 53, 54, 55];

(4) for linear preservers of $r_{\chi}(A)$ for special $\chi$, see [29, 33, 54].

(5) for linear preservers of $S p(A)$ or $\operatorname{Eig}(A)$, see [1, 26, 41.

Notice that if $\chi$ is of the determinant type, then

$$
|\operatorname{det}(A)|^{m / n}=\|K(A)\|=\rho(K(A))=r(K(A))=r_{\chi}(A)
$$

and

$$
\left\{\operatorname{det}(A)^{m / n}\right\}=W(K(A))=W_{\chi}(A)=S p(K(A))=\operatorname{Eig}(K(A)) .
$$

Using the results in 14,33 , one easily deduces the following.

Theorem 4.1. Suppose $\chi$ is of the determinant type. Let $F(A)=\|K(A)\|$, $\rho(K(A)), r(K(A)), r_{\chi}(A), W(K(A)), W_{\chi}(A), S p(K(A))$ or Eig $(K(A))$. A linear operator $L$ on $M_{n}$ satisfies

$$
F(L(A))=F(A) \quad \text { for all } A \in M_{n}
$$

if and only if there exists $M, N \in M_{n}$ with

(i) $|\operatorname{det}(M N)|=1$ if $F(A)=\|K(A)\|, \rho(K(A)), r(K(A))$ or $r_{\chi}(A)$,

(ii) $\operatorname{det}(M N)^{m / n}=1$ if $F(A)=W(K(A)), W_{\chi}(A), S p(K(A))$ or $\operatorname{Eig}(K(A))$, such that $L$ is of the form

$$
A \mapsto M A N \quad \text { or } \quad A \mapsto M A^{t} N .
$$

In the rest of this chapter, we focus on the case when $\chi$ is not of the determinant type. Some preliminary lemmas will be presented in the next section. We then characterize the linear preservers of $\|K(A)\|, \rho(K(A)), S p(K(A))$ and $\operatorname{Eig}(K(A))$ in $\S 4.2$. The rest of the chapter is devoted to studying the linear preservers of $W(K(A)), W_{\chi}(A), r(K(A))$ and $r_{\chi}(A)$.

\subsection{Preliminary Lemmas.}

Lemma 4.2. Suppose $\chi$ is the principal character. Let $A \in M_{n}$. Then

$$
\rho(K(A))=\rho(A)^{m} \leq r(A)^{m} \leq r_{\chi}(A) \leq r(K(A)) \leq\|K(A)\|=\|A\|^{m} .
$$

If $A$ is normal, then all the inequalities become equalities.

Proof. The first and last equality follows from the relations between the eigenvalues (respectively, singular values) of $A$ and $K(A)$ described in 1.4 (f) and the fact that there exists $\alpha \in \bar{\Delta}$ such that $m_{1}(\alpha)=m$.

Suppose $x \in \mathbb{C}$ is a unit vector such that $(A x, x)=z$ with $|z|=r(A)$. Let $x^{*}=x * \cdots * x$. Then $\left(K(A) x^{*}, x^{*}\right) /\left(x^{*}, x^{*}\right)=z^{m}$. Thus $r(A)^{m} \leq r_{\chi}(A)$. The other inequalities follow from Propositions 3.9 and 3.10 .

Clearly, if $A$ is normal, then so is $K(A)$. By Proposition 3.9, we have $\rho(K(A))=$ $\|K(A)\|$ and thus all the inequalities in (8) become equalities.

Lemma 4.3. Let $F(A)=\|K(A)\|, r(K(A)), r_{\chi}(A), \rho(K(A))$. Linear preservers of $F$ are invertible.

Proof. Let $L$ be a linear preserver of $F(A)=\|K(A)\|, r(K(A)), r_{\chi}(A), \rho(K(A))$. Suppose it is not invertible and $L(A)=0$ for some $A \neq 0$. Let $X, Y \in M_{n}$ be 
unitary such that $X^{*} A Y^{*}=\operatorname{diag}\left(a_{1}, \ldots, a_{n}\right)$ with $a_{1} \geq \cdots \geq a_{n} \geq 0$. Suppose $F(A)=\|K(A)\|, r(K(A))$ or $r_{\chi}(A)$. By Theorem 3.11 we have

$$
\begin{aligned}
1 & =\|K(X Y)\|=\|K(L(X Y))\| \\
& =\|K(L(X Y+A))\|=\|K(X Y+A)\| \\
& \geq|\operatorname{det}(X Y+A)|^{m / n}=\left|\operatorname{det}\left(I+X^{*} A Y^{*}\right)\right|^{m / n} \\
& =\left|\prod_{j=1}^{n}\left(1+a_{j}\right)\right|^{m / n}>1,
\end{aligned}
$$

which is a contradiction.

If $F(A)=\rho(K(A))$, we can find a nilpotent matrix $N$ so that $A+N$ is nonsingular. It follows that

$$
0 \neq \rho(K(A+N))=\rho(K(L(A+N)))=\rho(K(L(N)))=\rho(K(N))=0,
$$

which is a contradiction.

4.2. Invariance of Spectral Norm and Spectral Radius. In this section, we consider linear preservers of

$$
\|K(A)\|, \quad \rho(K(A)), \quad S p(K(A)) \text { and } \operatorname{Eig}(K(A)) .
$$

Theorem 4.4. Suppose $\chi$ is not of the determinant type. A linear operator $L$ on $M_{n}$ satisfies

$$
\|K(L(A))\|=\|K(A)\| \quad \text { for all } A \in M_{n}
$$

if and only if there exist unitary $U, V \in M_{n}$ such that $L$ is of the form

$$
A \mapsto U A V \quad \text { or } \quad A \mapsto U A^{t} V .
$$

Proof. The sufficiency part is clear. We prove the converse below.

If $\chi$ is the principal character, then $\|K(A)\|=\|A\|^{m}$ by Lemma 4.2. Hence, if $\|K(A)\|=\|K(L(A))\|$ for all $A \in M_{n}$, then $\|A\|=\|L(A)\|$ for all $A \in M_{n}$. By the result in [27] (see also [15] or [34]), $L$ is of the asserted form.

Suppose $\chi$ is not the principal character. By Lemma 4.3, $L$ is invertible. Let

$$
R=\left\{A \in M_{n}:\|K(A)\|=0\right\} .
$$

Then clearly, $L(R) \subseteq R$. Suppose $r=\mu(\bar{\Delta})$. By the result in [9], we have $r>1$ and $R$ is the set of matrices with rank at most $r-1$. By the result in [3], $L$ is of the form

$$
\text { (i) } A \mapsto M A N \quad \text { or } \quad \text { (ii) } A \mapsto M A^{t} N \text {, }
$$

for some invertible $M, N \in M_{n}$.

Suppose $U_{1} U_{2}, V_{1}, V_{2} \in M_{n}$ are unitary such that

$$
U_{1} M U_{2}=\operatorname{diag}\left(\xi_{1}, \ldots, \xi_{n}\right) \text { and } V_{1} N V_{2}=\operatorname{diag}\left(\eta_{1}, \ldots, \eta_{n}\right)
$$

with $\xi_{1} \geq \cdots \geq \xi_{n}>0$ and $\eta_{1} \geq \cdots \geq \eta_{n}>0$. We claim that $\xi_{1}=\xi_{n}$ and $\eta_{1}=\eta_{n}$. If it is not true, then $\xi_{1} \eta_{1}>\xi_{n} \eta_{n}$. Consider $A, B \in M_{n}$ such that

$$
A^{+}=U_{2} \operatorname{diag}\left(1 /\left(\xi_{n} \eta_{n}\right), \ldots, 1 /\left(\xi_{1} \eta_{1}\right)\right) V_{1}
$$

and

$$
B^{+}=U_{2} \operatorname{diag}\left(1 /\left(\xi_{1} \eta_{1}\right), \ldots, 1 /\left(\xi_{n} \eta_{n}\right)\right) V_{1},
$$


where

$$
X^{+}= \begin{cases}X & \text { if (i) holds, } \\ X^{t} & \text { if (ii) holds. }\end{cases}
$$

Then $A$ and $B$ have the same singular values and hence $\|K(A)\|=\|K(B)\|$. However, $L(B)$ is unitary and thus $\|K(L(B))\|=1$, whereas $L(A)$ has singular values

$$
\left(\xi_{1} \eta_{1}\right) /\left(\xi_{n} \eta_{n}\right) \geq \cdots \geq\left(\xi_{n} \eta_{n}\right) /\left(\xi_{1} \eta_{1}\right)
$$

with $\left(\xi_{1} \eta_{1}\right) /\left(\xi_{n} \eta_{n}\right)>\left(\xi_{n} \eta_{n}\right) /\left(\xi_{1} \eta_{1}\right)$ so that $L(A)$ is not unitary. Since $\chi$ is not of the determinant type, by Theorem 3.11 we have

$$
\|K(A)\|=\|K(L(A))\|>|\operatorname{det}(L(A))|^{m / n}=1=\|K(B)\|,
$$

which is a contradiction.

Now, since $1=\|K(I)\|=\|K(L(I))\|$, we see that $\left|\xi_{j} \eta_{j}\right|=1$ for all $j$. Hence, $L$ is of the asserted form with $U=M / \xi_{1}$ and $V=N / \eta_{1}$.

Theorem 4.5. Suppose $\chi$ is not of the determinant type. A linear operator $L$ on $M_{n}$ satisfies

$$
\rho\left(K(L(A))=\rho(K(A)) \quad \text { for all } A \in M_{n}\right.
$$

if and only if there exist an invertible $S \in M_{n}$ and some $\xi \in \mathbb{C}$ with $|\xi|=1$ such that $L$ is of the form

$$
A \mapsto \xi S^{-1} A S \quad \text { or } \quad A \mapsto \xi S^{-1} A^{t} S .
$$

Proof. The sufficiency part is clear. We prove the converse below.

If $\chi$ is the principal character, then $\rho(K(A))=\rho(A)^{m}$ by Lemma 4.5 Hence, a linear preserver of $\rho(K(A))$ is also a linear preserver of $\rho(A)$. By the result in [8] or [32], $L$ is of the asserted form.

Suppose $\chi$ is not the principal character. By Lemma 4.3, $L$ is invertible. Let

$$
R=\left\{A \in M_{n}: \rho(K(A))=0\right\} .
$$

Then clearly, $L(R) \subseteq R$. Suppose $r=\mu(\bar{\Delta})$. By the result in [9], we have $r \geq 2$. By Proposition $1.4(\mathrm{~h}), R$ is the set of matrices with at most $r-1$ nonzero eigenvalues, i.e., at least $n-r+1$ zero eigenvalues. If $2 \leq n-r+1 \leq n-1$, i.e., $n-1 \geq r \geq 2$, then $L$ is of the asserted form by the result in [31].

Now, suppose $r=n$. Then $R$ is the set of singular matrices. Since $L$ is invertible and $L(R) \subseteq R$, by the result in [11] (see also [3]), we see that $L$ is of the form

$$
\text { (i) } A \mapsto M A N \quad \text { or } \quad \text { (ii) } A \mapsto M A^{t} N \text {, }
$$

for some invertible $M, N \in M_{n}$. We claim that $M N=\xi I$. Suppose that it is not true. If $M N$ is not diagonalizable, then $M N$ has a nontrivial Jordan block in its Jordan form; if $M N$ is diagonalizable, then $M N$ is similar to $\operatorname{diag}\left(\lambda_{1}, \ldots, \lambda_{n}\right)$ with $\lambda_{1} \neq \lambda_{2}$, and thus similar to $\operatorname{diag}\left(\lambda_{1}, \ldots, \lambda_{n}\right)+E_{12}$. In both cases, there exists an invertible $T \in M_{n}$ such that $M N=T^{-1} X T$, where $X$ is in upper triangular form with diagonal entries $\lambda_{1}, \ldots, \lambda_{n}$ and $(1,2)$ entry equal to 1 . Let

$$
Z^{+}= \begin{cases}Z & \text { if (i) holds } \\ Z^{t} & \text { if (ii) holds. }\end{cases}
$$

For $t>0$, consider the matrix $A(t) \in M_{n}$ such that

$$
A(t)^{+}=N T^{-1}\left(I+t E_{21}\right) T N^{-1} .
$$


Then $L(A(t))$ is similar to the matrix

$$
X(t)=X+t E_{11}+t \lambda_{2} E_{21}
$$

Since $\chi$ is not of the determinant type, there exits $\alpha \in \bar{\Delta}$ such that $m_{1}(\alpha)>m_{n}(\alpha)$ and $\rho(K(B))=\prod_{j=1}^{n}\left|\eta_{j}^{m_{j}(\alpha)}\right|$ whenever $B \in M_{n}$ has eigenvalues $\eta_{1}, \ldots, \eta_{n}$ with $\left|\eta_{1}\right| \geq \cdots \geq\left|\eta_{n}\right|$. By this fact, one can see that $\rho(K(X(t))) \rightarrow \infty$ as $t \rightarrow \infty$. This contradicts the fact that

$$
1=\rho(K(A(t)))=\rho(K(L(A(t))))=\rho(K(X(t)))
$$

for all $t>0$. Thus, our claim is proved.

Finally, since $1=\rho(K(I))=\rho(K(L(I)))=\left|\xi^{m}\right|$, we see that $|\xi|=1$. Hence $L$ is of the asserted form.

An easy consequence of Theorem 4.5 is the following result.

Corollary 4.6. Suppose $\chi$ is not of the determinant type, and

$$
F(A)=S p(K(A)) \text { or } \operatorname{Eig}(K(A)) \text { on } M_{n} \text {. }
$$

A linear operator $L$ on $M_{n}$ satisfies

$$
F(L(A))=F(A) \quad \text { for all } A \in M_{n}
$$

if and only if there exist an invertible $S \in M_{n}$ and some $\xi \in \mathbb{C}$ with $\xi^{m}=1$ such that $L$ is of the form

$$
A \mapsto \xi S^{-1} A S \quad \text { or } \quad A \mapsto \xi S^{-1} A^{t} S .
$$

4.3. Invariance of Numerical Ranges and Radii. In this section, we consider the linear preservers of the function

$$
F(A)=W(K(A)), W_{\chi}(A), r(K(A)) \text { or } r_{\chi}(A) .
$$

First of all, we can use the results in the previous chapters to prove the following.

Theorem 4.7. Suppose $\chi$ is not of the determinant type. Let

$$
F(A)=W(K(A)) \text { or } W_{\chi}(A) \text { on } M_{n} \text {. }
$$

A linear operator $L$ on $M_{n}$ satisfies

$$
F(L(A))=F(A) \quad \text { for all } A \in M_{n}
$$

if and only if there exist a unitary $U \in M_{n}$ and $a \xi \in \mathbb{C}$ with $\xi^{m}=1$ such that $L$ is of the form

$$
A \mapsto \xi U^{*} A U \quad \text { or } \quad A \mapsto \xi U^{*} A^{t} U .
$$

Proof. We focus on the case $F(A)=W_{\chi}(A)$. The case of $F(A)=W(K(A))$ can be proved by a similar and simpler argument.

The sufficiency part is clear. To prove the converse, let $L$ be a linear preserver of $W_{\chi}$. Since $W_{\chi}(L(I))=W_{\chi}(I)=\{1\}$, by Theorem 3.7(b) we see that $L(I)=\xi I$ for some $\xi \in \mathbb{C}$ with $\xi^{m}=1$. For simplicity, assume that $L(I)=I$. Otherwise, replace $L$ by $L / \xi$. If $A$ is positive definite, then, by Corollary [3.6] we have $W_{\chi}(L(I+t A))=$ $W_{\chi}(I+t A) \subseteq(0, \infty)$ for all $t>0$, and hence $L(A)$ is a multiple of a positive definite matrix. We see that $L$ maps the set of positive definite matrices into itself.

Note that if $L$ preserves $W_{\chi}(A)$, then it also preserves $r_{\chi}(A)$. By Lemma 4.3 $L$ is invertible. It is easy to check check that $L^{-1}$ also preserves $W_{\chi}(A)$. So, one can apply the previous arguments to $L^{-1}$ to conclude that $L^{-1}$ maps the set of positive 
definite matrices into itself. Thus $L$ maps the set of positive definite matrices onto itself. By continuity, $L$ maps the set of positive semi-definite matrices onto itself. We can then apply a result of Schneider [51] to conclude that $L$ is of the form

$$
A \mapsto U^{*} A U \quad \text { or } \quad A \mapsto U^{*} A^{t} U
$$

for some invertible $U \in M_{n}$. Since $L(I)=I$, we conclude that $U^{*} U=I$.

In the study of linear preserver problems, it is often the case that linear preservers of the generalized numerical radii are unit multiples of linear preservers of the corresponding numerical ranges (see [46, Chapter 6]). However, the proofs of such results are usually very involved. We have a similar phenomenon for linear preservers of $F(A)=r(K(A))$ or $r_{\chi}(A)$. In particular, we have the following result.

Theorem 4.8. Suppose $\chi$ is not of the determinant type. Let

$$
F(A)=r(K(A)) \text { or } r_{\chi}(A) \text { on } M_{n} .
$$

A linear operator $L$ on $M_{n}$ satisfies

$$
F(L(A))=F(A) \quad \text { for all } A \in M_{n}
$$

if and only if there exist a unitary $U \in M_{n}$ and $a \xi \in \mathbb{C}$ with $|\xi|=1$ such that $L$ is of the form

$$
A \mapsto \xi U^{*} A U \quad \text { or } \quad A \mapsto \xi U^{*} A^{t} U .
$$

The proof of this result will be done by a group theory scheme (see $\S 4.4)$ and completed in $\S 4.5$. Here we use Theorem 4.8 to give a short proof of Theorem 4.7 as follows.

The sufficiency part is clear. To prove the converse, note that if $L$ preserves $W(K(A))$, then $L$ preserves $r(K(A))$; if $L$ preserves $W_{\chi}(A)$, then $L$ preserves $r_{\chi}(A)$. Thus, $L$ is of the form in Theorem 4.8 It follows that $\left\{\xi^{m}\right\}=F(L(I))=F(I)=$ $\{1\}$, and hence $\xi^{m}=1$.

4.4. A Group Scheme. Dynkin [13] used a group scheme to study linear preserver problems. A similar scheme has been carried out by several authors recently (see 12, 16, 17, 48, 49] and their references). We shall use a similar method in our study.

Suppose $P S U(n)$ is the group of operators on $M_{n}$ of the form

$$
A \mapsto U^{*} A U
$$

for some unitary $U \in M_{n}$. We shall prove that linear preservers of $F(A)=r_{\chi}(A)$ or $r(K(A))$ form a group $G$. Clearly, every element in $P S U(n)$ is a linear preserver of $F$. Hence $P S U(n)$ is a subgroup of $G$. Let $G_{0}$ be the largest connected Lie subgroup in $G$. Using the results in [49] (see also [17]) and those in the previous sections, we will show that $G_{0}=P S U(n)$, and then completely determine $G$.

Here we introduce some notations and list the group theory results that will be used in our proof.

Let $G L(n), S L(n), U(n)$ and $S U(n)$ be the general linear group, special linear group, unitary group, and special unitary group of linear operators acting on $\mathbb{C}^{n}$. Similarly, let $G L\left(n^{2}\right), S L\left(n^{2}\right)$ and $S U\left(n^{2}\right)$ be the general linear group, special linear group and special unitary group of operators acting on $M_{n}$, respectively.

Denote by $S U(n) * S U(n)$ the group of operators of the form $A \mapsto U A V$ for some $U, V \in S U(n)$. Moreover, let $S O\left(n^{2}\right)$ be the subgroup of $S U\left(n^{2}\right)$ consisting 
of operators mapping the real linear space of hermitian matrices onto itself. Thus, $S O\left(n^{2}\right)$ can be viewed as the complex extension of those (real) linear operators on the space of hermitian matrices preserving the inner product $(A, B)=\operatorname{tr}(A B)$.

Let $G L\left(n^{2}-1\right)$ be the subgroup of $G L\left(n^{2}\right)$ consisting of operators that fix the identity and map the subspace $M_{n}^{\prime}$ of matrices with zero trace onto itself, and let

$$
S U\left(n^{2}-1\right)=S U\left(n^{2}\right) \cap G L\left(n^{2}-1\right) \text { and } S O\left(n^{2}-1\right)=S O\left(n^{2}\right) \cap G L\left(n^{2}-1\right) .
$$

Furthermore, let $\mathbf{T}$ be the group of operators acting as scalar on $M_{n}^{\prime}$ and $\operatorname{span}\{I\}$, and let $\mathbf{U}_{1}$ be the intersection of $\mathbf{T}$ and $S U\left(M_{n}\right)$.

When $n=4$, we have a special subgroup $\Lambda_{0}$, an embedding of $S U(6) /\langle-1\rangle$ in $S U\left(4^{2}-1\right)$, containing $P S U(4)$. We refer the readers to [49] for a concrete construction and some discussion of $\Lambda_{0}$. For our purpose, we only need to know (see 49, p. 151]) that $\Lambda_{0}$ contains an operator mapping $\operatorname{diag}(1,1,-1,-1)$ to $2 E_{13} \in M_{4}^{\prime}$.

With the above notations, we are ready to state the following results [49], Theorem 1] and [49, Theorems 3 and 4 (ii)] (see also [17]).

Proposition 4.9. Suppose $G_{0}$ is a connected compact subgroup of $S L\left(M_{n}\right)$ containing $P S U(n)$. If $G_{0}$ is reducible, then $G_{0}=H_{0}$ or $H_{0} \mathbf{U}_{1}$ where

$$
H_{0}=P S U(n), S O\left(n^{2}-1\right), S U\left(n^{2}-1\right), \quad \text { or } \Lambda_{0}(n=4) .
$$

If $G_{0}$ is irreducible, then it is a $\mathbf{T}$-conjugate of one of the following groups:

$$
S U(n) * S U(n), S O\left(n^{2}\right), S U\left(n^{2}\right) .
$$

Proposition 4.10. Let $G$ be a subgroup of $G L\left(n^{2}\right)$ containing $P S U(n)$ as the largest connected Lie subgroup. Then $G$ is a subgroup of the group generated by $P S U(n), \mathbf{T}$ and the transposition operator $A \mapsto A^{t}$.

4.5. Proof of Theorem 4.8. Let $\chi$ and $F$ satisfy the hypotheses of the theorem. The sufficiency part is clear. We prove the converse below.

First of all, we establish the following

Assertion. The linear preservers of $F(A)=r_{\chi}(A)$ or $r(K(A))$ on $M_{n}$ form a compact group $G$ in $G L\left(n^{2}\right)$. Moreover, if $\chi$ is not the principal character, then $G$ is a subgroup of $S U(n) * S U(n)$.

Suppose $L$ is a linear preserver of $F$. By Lemma 4.3 If $L$ preserves $F$, one easily checks that $L^{-1}$ also preserves $F$. Thus the linear preservers of $F$ form a group $G$ of invertible operators on $M_{n}$. Clearly, the limit of a convergent sequence of linear preservers of $F$ is also a preserver of $F$, hence $G$ is closed.

Now, suppose $\chi$ is the principal character. Let $L \in G$. By Lemma 4.2 for any unitary $U$ we have

$$
1=F(U)=F(L(U)) \geq r(L(U))^{m} .
$$

Thus, the set of unitary matrices will be mapped to a bounded set under a linear preserver of $F$. Consequently, $G$ is bounded. Thus, the group $G$ is compact.

Next, suppose $\chi$ is not the principal character. Let $r=\mu(\bar{\Delta})$. By the result in 9, we have $r>1$, and $F(A)=0$ if and only if $\operatorname{rank}(A)<r$. Hence, if $L$ is a preserver of $F$, then $L$ maps the set of matrices with rank at least $r$ to itself. By the result in [3], there exist invertible $M, N \in M_{n}$ with $\operatorname{det}(M N)=1$ such that $L$ is of the form

$$
A \mapsto M A N \quad \text { or } \quad A \mapsto M A^{t} N
$$


By Theorem 3.11 ,

$$
1=F(I)^{n}=F(L(I))^{n} \geq|\operatorname{det}(L(I))|^{m}=|\operatorname{det}(M N)|^{m}
$$

and

$$
1=F(I)^{n}=F\left(L^{-1}(I)\right)^{n} \geq\left|\operatorname{det}\left(L^{-1}(I)\right)\right|^{m}=\left|\operatorname{det}\left(M^{-1} N^{-1}\right)\right|^{m} .
$$

It follows that $|\operatorname{det}(M N)|=1$, and hence

$$
\operatorname{det}(L(A))=\operatorname{det}(A) \quad \text { for all } A \in M_{n} .
$$

As a result, if $A \in M_{n}$ is unitary, then

$$
|\operatorname{det}(L(A))|=|\operatorname{det}(A)|=1=F(A)=F(L(A)) \text {. }
$$

It follows from Theorem 3.11 (b) that $L(A)$ is unitary. Thus, $L$ maps unitary matrices to unitary matrices. By the result in [35], we see that $G$ is a subgroup of $S U(n) * S U(n)$. The proof of the Assertion is complete.

Let $G_{0}$ be the largest connected Lie group contained in $G$. Then $G_{0}$ must be one of the groups listed in Proposition 4.9. We shall prove that $G_{0}=P S U(n)$ in the following steps. In each step, we show that for any group in Proposition 4.9 not equal to $P S U(n)$, there are some linear operators $L$ in it and $A \in M_{n}$ such that $F(A) \neq F(L(A))$. For nonprincipal characters, sometimes we just use the fact that every linear preserver $L \in G_{0}$ satisfies (9) to get the desired conclusion.

Step 1. $G_{0}$ is not a $\mathbf{T}$-conjugate of $S U(n) * S U(n)$.

Suppose to the contrary that there exists $S \in \mathbf{T}$ of the form $A \mapsto a(\operatorname{tr} A) I / n+$ $b(A-(\operatorname{tr} A) I / n)$ such that $S^{-1} G_{0} S=S U(n) * S U(n)$. We may assume that $b=1$; otherwise, replace $S$ by $S / b$.

If $n>2$, let $A=n E_{11}-I_{n}$. Then there exists $L \in S U(n) * S U(n)$ such that

$$
S^{-1} L S(A)=B=\sum_{j=1}^{n-1} E_{j, j+1}+(n-1) E_{n 1} .
$$

By Proposition 3.10 (b), $\|K(A)\|=F(A)$. However, all eigenvalues of $B$ are the same, and $B$ is not normal. By Corollary 3.12 , we see that

$$
F(B)<\|K(B)\|=\|K(A)\|=F(A),
$$

which is a contradiction.

Suppose $n=2$. If $\chi$ is not the principal character, then $G_{0} \subseteq S U(2) * S U(2)$. If $G_{0}$ is also a $\mathbf{T}$-conjugate of $S U(2) * S U(2)$, we must have $G_{0}=S U(2) * S U(2)$. There exists $L \in S U(2) * S U(2)$ such that for $A=2 E_{11}+E_{22}$, we have $L(A)=$ $B=2 E_{12}+E_{21}$. Now, all eigenvalues of $B$ have the same magnitude and $B$ is not normal. By Corollary 3.12, we have

$$
F(B)<\|K(B)\|=\|K(A)\|=F(A)
$$

which is a contradiction.

Now, suppose $\chi$ is the principal character. Let $L \in S U(2) * S U(2)$ be such that $L(X)=X\left(E_{11}-E_{22}\right)$ and let $\tilde{L}(X)=\left(S^{-1} L S\right)(X)$ for some $S \in \mathbf{T}$. Then $\tilde{L}(I)=$ $a E_{11}-a E_{22}$. Since $F(\tilde{L}(I))=F(I)=1$, we see that $|a|=1$. If $A=\left(\begin{array}{ll}1 & 1 \\ 1 & 1\end{array}\right)$, then 
$\tilde{L}(A)=B=\left(\begin{array}{ll}a & -1 \\ 1 & -a\end{array}\right)$. Since $\operatorname{tr} B=0$, the eigenvalues of $B$ are of the form $\pm z$. If $B$ is normal, then $2|z|^{2}=\operatorname{tr} B B^{*}=4$. It follows that

$$
F(B)=|z|^{m}=2^{m / 2}<2^{m}=F(A) .
$$

If $B$ is not normal, then by Corollary 3.12 we have

$$
F(B)<\|K(B)\| \leq\left(\operatorname{tr} B B^{*}\right)^{m / 2}=2^{m}=F(A) .
$$

In both cases, we get the desired contradiction.

Step 2. $G_{0}$ does not contain $\Lambda_{0}$.

Suppose to the contrary that $G_{0}$ contains $\Lambda_{0}$. Then (see [49, p. 151]) there is a linear preserver $L$ of $F$ such that

$$
L(A)=2 E_{13} \text { with } A=\operatorname{diag}(1,1,-1,-1) \text { and } L(I)=I .
$$

If $\chi$ is not the principal character, then $|\operatorname{det}(L(A))|=0 \neq 1=\mid \operatorname{det}(A)) \mid$, contradicting (9).

If $\chi$ is the principal character, then by Lemma 4.2 we have

$$
F(I+i A)=\|I+i A\|^{m}=2^{m / 2}<2^{m}=[r(L(I+i A))]^{m} \leq F(L(I+i A)),
$$

which is a contradiction.

Step 3. $n>2$ and $G_{0}$ does not contain $S O\left(n^{2}-1\right), S U\left(n^{2}-1\right)$ or a T-conjugate of $S O\left(n^{2}\right)$ and $S U\left(n^{2}\right)$.

Since $S O\left(n^{2}-1\right)$ is a subgroup of $S U\left(n^{2}-1\right)$ and of any T-conjugate of $S O\left(n^{2}\right)$ and $S U\left(n^{2}\right)$, it is enough to show that $G_{0}$ does not contain $S O\left(n^{2}-1\right)$. Note that $S O\left(2^{2}-1\right)$ is the same as $P S U(2)$. That is why we impose the assumption that $n>2$.

Suppose to the contrary that $G_{0}$ contains $S O\left(n^{2}-1\right)$ with $n>2$. Then there is a linear preserver $L$ of $F$ such that $L(A)=\sqrt{n(n-1) / 2}\left(E_{11}-E_{22}\right)$ with $A=n E_{11}-$ $I_{n}$. If $\chi$ is not the principal character, then $|\operatorname{det}(A)|=n-1 \neq 0=|\operatorname{det}(L(A))|$, contradicting (9). If $\chi$ is the principal character, then by Lemma 4.2 we have

$$
F(A)=(n-1)^{m}>(n(n-1) / 2)^{m / 2}=F(L(A)),
$$

which is a contradiction.

Step 4. $n=2$ and $G_{0}$ does not contain $S U\left(2^{2}-1\right)$ or a $\mathbf{T}$-conjugate of $S U\left(2^{2}\right)$.

Since $S U\left(2^{2}-1\right)$ is a subgroup of any $\mathbf{T}$-conjugate of $S U\left(2^{2}\right)$, it is enough to show that $G_{0}$ does not contain $S U\left(2^{2}-1\right)$.

Suppose $G_{0}$ indeed contains $S U\left(2^{2}-1\right)$. Then there is a linear preserver $L$ of $F$ such that $L(I)=I$ and $L(A)=i A$ for $A=E_{11}-E_{22}$. If $\chi$ is not the principal character, then $|\operatorname{det}(I+A)|=0 \neq 2=|\operatorname{det}(L(I+A))|$, contradicting (99). If $\chi$ is the principal character, then by Lemma 4.2,

$$
F(I+A)=2^{m}>2^{m / 2}=F(L(I+A)),
$$

which is a contradiction.

Step 5. $n=2$ and $G_{0}$ does not contain a T-conjugate of $S O\left(2^{2}\right)$.

Suppose there exists $S \in \mathbf{T}$ of the form $A \mapsto a(\operatorname{tr} A) I / 2+b(A-(\operatorname{tr} A) I / 2)$ such that $S^{-1} G_{0} S=S O\left(2^{2}\right)$. We may assume that $b=1$; otherwise, replace $S$ by $S / b$.

If $\chi$ is not the principal character, then $G_{0}$ is a subgroup of $S U(2) * S U(2)$. By Step 1 , it is a proper subgroup, and hence the real dimension of $G_{0}$ is strictly less 
than that of $S U(2) * S U(2)$, which is 6 . On the other hand, the real dimension of $S O\left(2^{2}\right)$ is 6 . Thus, it is impossible for $G_{0}$ to be a $\mathbf{T}$-conjugate of $S O\left(2^{2}\right)$.

Next, suppose $\chi$ is the principal character. Let $L \in S O\left(2^{2}\right)$ be such that $L$ maps the orthogonal pair of matrices $\left(I, E_{12}+E_{21}\right)$ to the pair $\left(E_{11}-E_{22}, E_{12}+E_{21}\right)$, and let $\tilde{L}(X)=S^{-1} L S(X)$. Since $\tilde{L}(I)=a\left(E_{11}-E_{22}\right)$ and $F(\tilde{L}(I))=F(I)=1$, we see that $|a|=1$. If $A=\left(\begin{array}{ll}1 & 1 \\ 1 & 1\end{array}\right)$, then $\tilde{L}(A)=B=\left(\begin{array}{cc}a & 1 \\ 1 & -a\end{array}\right)$. Since $\operatorname{tr} B=0$, the eigenvalues of $B$ are of the form $\pm z$. If $B$ is normal, then $2|z|^{2}=\operatorname{tr} B B^{*}=4$. It follows that

$$
F(B)=|z|^{m}=2^{m / 2}<2^{m}=F(A) .
$$

If $B$ is not normal, then by Corollary 3.12 we have

$$
F(B)<\|K(B)\| \leq\left(\operatorname{tr} B B^{*}\right)^{m / 2}=2^{m}=F(A) .
$$

In both cases, we get the desired contradiction.

Step 6. $G \cap \mathbf{T}$ is the circle group, i.e., group of operators of the form $A \mapsto a A$, where $a \in \mathbb{C}$ satisfies $|a|=1$. In particular, $\mathbf{U}_{1}$ is not a subgroup of $G_{0}$.

Suppose $L \in \mathbf{T}$ is a linear preserver of $F$ such that

$$
L(A)=a(\operatorname{tr} A) I / n+b(A-(\operatorname{tr} A) I / n), \quad a, b \in \mathbb{C} .
$$

Since $F(I)=F(L(I))$, we conclude that $|a|=1$. We may assume that $a=1$ and hence $L(I)=I$. Otherwise, replace $L$ by $a^{-1} L$. Now, $L\left(E_{11}\right)=(1-b) I / n+b E_{11}$. Suppose $b \neq 1$. If $\chi$ is not the principal character, then it is impossible to have

$$
\begin{aligned}
z^{n-1}(z+1) & =\operatorname{det}\left(z I+E_{11}\right) \\
& =\operatorname{det}\left(L\left(z I+E_{11}\right)\right) \\
& =\left(z+\frac{1-b}{n}+b\right)\left(z+\frac{1-b}{n}\right)^{n-1}
\end{aligned}
$$

for all $z \in \mathbb{C}$, contradicting (9). If $\chi$ is the principal character, then one can check that it is impossible to have

$$
\begin{aligned}
\max \left\{|z+1|^{m},|z|^{m}\right\} & =F\left(z I+E_{11}\right) \\
& =F\left(L\left(z I+E_{11}\right)\right) \\
& =\max \left\{|z+b+(1-b) / n|^{m},|z+(1-b) / n|^{m}\right\}
\end{aligned}
$$

for all $z \in \mathbb{C}$. In both cases, we must have $b=1$ and hence $L(A)=A$.

We are now ready to complete the proof of Theorem 4.8: By Steps 1-6, we see that $G_{0}=P S U(n)$ for any $\chi$. By Proposition 4.10, $G$ is a subgroup of the group generated by $P S U(n), \tau$ and T. Clearly, $\tau \in G$. By Step 6 , we see that $G$ can only contain the circle group of $\mathbf{T}$. The result on $F$ preservers follows.

4.6. Results on Hermitian Matrices. One can get similar results for linear preservers of $F$ on hermitian matrices by simpler arguments. Note that $\rho(K(A))=$ $r_{\chi}(A)=r(K(A))=\|K(A)\|$ for hermitian matrices $A$. If $\chi$ is the principal character, then linear preservers of $r_{\chi}$ are just the linear preservers of the numerical radius by Lemma 4.2 and the structure of them is known (see [29]). If $\chi$ is not the principal character, then by the result in [9] we have $r=\mu(\bar{\Delta})>1$. Now, one can show that linear preservers of $r_{\chi}$ are invertible and they preserve matrices with rank less than $r$ by arguments similar to those in the proofs of Lemma 4.3 
and Theorem 4.5. Then one can apply the result in 21] to conclude that the linear preserver is of the form

$$
A \mapsto \xi S^{*} A S \quad \text { or } \quad A \mapsto \xi S^{*} A^{t} S
$$

for some invertible $S$ and $\xi= \pm 1$, and determine the structure of linear preservers of $r_{\chi}$. In summary, we have the following result.

Theorem 4.11. Let $F(A)=\rho(K(A))=r_{\chi}(A)=r(K(A))=\|K(A)\|$ on $n \times n$ hermitian matrices. Then a (real) linear operator $L$ on $n \times n$ hermitian matrices is a linear preserver of $F$ if and only if there is $\xi= \pm 1$ such that $L$ is of the form

$$
A \mapsto \xi S^{*} A S \quad \text { or } \quad A \mapsto \xi S^{*} A^{t} S,
$$

where $S \in S L(n)$ in case $\chi$ is of the determinant type and $S \in S U(n)$ otherwise.

Using the above theorem, one easily gets the following corollary.

Corollary 4.12. Let $F(A)=S p(K(A))$, Eig $(K(A)), W_{\chi}(A)$, or $W(K(A))$ on $n \times n$ hermitian matrices. Then a (real) linear operator $L$ on $n \times n$ hermitian matrices is a linear preserver of $F$ if and only if there is $\xi= \pm 1$ with $\xi^{m}=1$ such that $L$ is of the form

$$
A \mapsto \xi S^{*} A S \quad \text { or } \quad A \mapsto \xi S^{*} A^{t} S,
$$

where $S \in S L(n)$ in case $\chi$ is of the determinant type and $S \in S U(n)$ otherwise.

\section{ACKNOWLEDGEMENT}

Research of the first author was supported by an NSF grant of the USA. This research was done while he was visiting the University of Toronto and supported by a faculty research grant of the College of William and Mary in the academic year 1998-1999. He would like to thank Professor M.D. Choi for making the visit possible. The second author was supported by a grant of Professors E. Bierstone, A. Khovanskii, P. Milman and M. Spivakovsky of the University of Toronto.

Both authors would like to thank the staff of the University of Toronto for their warm hospitality. They would also like to thank Professors J.A. Dias da Silva, A. Fonseca, R. Merris, T. Pate and T.Y. Tam for some helpful correspondence.

\section{REFERENCES}

[1] P. Andresen and M. Marcus, Weyl's inequality and quadratic forms on the Grassmannian, Pacific J. Math. 67 (1976), 277-289. MR 55:2963

[2] B. Aupetit and H. du T. Mouton, Spectrum preserving linear mappings in Banach algebras, Studia Math. 109 (1994), 91-100. MR 95c:46070

[3] L. Beasley, Linear transformations on matrices: The invariance of sets of ranks, Linear Algebra Appl. 48 (1982), 25-35. MR 84e:15001

[4] N. Bebiano and C.K. Li, A brief survey on the decomposable numerical range of matrices, Linear and Multilinear Algebra 32 (1992), 179-190. MR 94g:15019

[5] N. Bebiano, C.K. Li and J. da Providência, The numerical range and decomposable numerical range of matrices, Linear and Multilinear Algebra 29 (1991), 195-205. MR 94g:15016

[6] N. Bebiano, C.K. Li and J. da Providência, Some results on the numerical range of a derivation, SIAM J. Matrix Analysis Appl. 14 (1993), 1084-1095. MR 94i:15022

[7] N. Bebiano, C.K. Li and J. da Providência, Generalized Numerical Ranges of Permanental Compounds Arising From Quantum Systems of Bosons, Electron J. Linear Algebra 7 (2000), 73-91. CMP 2000:16

[8] M. Brešar and P. Šemrl, Linear maps preserving the spectral radius, J. Functional Analysis 142 (1996), 360-368. MR 97i:47070 
[9] C.F. Chan, Some more on a conjecture of Marcus and Wang, Linear and Multilinear Algebra 25 (1989), 231-235. MR 90h:20014

[10] J.A. Dias da Silva and A. Fonseca, Nonzero star products, Linear and Multilinear Algebra 27 (1990), 49-55. MR 91h:15030

[11] J. Dieudonné, Sur une Generalisation du groupe orthogonal à quatre variables, Arch. Math 1 (1949), 282-287. MR 10:5861

[12] D.Z. Doković and C.K. Li, Overgroups of some classical linear groups with applications to linear preserver problems. Second Conference of the International Linear Algebra Society (ILAS) (Lisbon, 1992). Linear Algebra Appl. 197/198 (1994), 31-61. MR 95i:20069

[13] E.B. Dynkin, The maximal subgroups of the classical groups, Amer. Math. Soc. Transl. Ser. 6 (1957), 245-378.

[14] G. Frobenius, Uber die Darstellung der endichen Gruppen durch Linear Substitutionen, S. B. Deutsch. Akad. Wiss. Berlin (1897), 994-1015.

[15] R. Grone and M. Marcus, Isometries of matrix algebras, J. Algebra 47 (1977), 180-189. MR 56:3039

[16] R.M. Guralnick, Invertible preservers and algebraic groups. II. Preservers of similarity invariants and overgroups of $\mathrm{PSL}_{n}(\mathbb{F})$. Linear and Multilinear Algebra 43 (1997), 221-255. MR 99m:20108

[17] R.M. Guralnick and C.K. Li, Invertible preservers and algebraic groups III: Preservers of unitary similarity (congruence) invariants and overgroups of some unitary groups, Linear and Multilinear Algebra 43 (1997), 257-282. MR 99m:20109

[18] W. Greub, Multilinear Algebra, 2nd ed., Springer-Verlag, New York, 1978. MR 80c:15017

[19] K.R. Gustafson and D.K.M. Rao, Numerical range: The field of values of linear operators and matrices, Universitext, Springer-Verlag, New York, 1997. MR 98b:47008

[20] P.R. Halmos, A Hilbert Space Problem Book, Second Ed., Springer-Verlag, New York, 1982. MR 84e:47001

[21] J.W. Helton and L. Rodman, Signature preserving linear maps of hermitian matrices, Linear and Multilinear Algebra 17 (1985), 29-37. MR 87m:15055

[22] A. Horn, On the eigenvalues of a matrix with prescribed singular values, Proc. Amer. Math. Soc. 5 (1954), 4-7. MR 15:847d

[23] R.A. Horn and C.R. Johnson, Topics in Matrix Analysis, Cambridge University Press, Cambridge, 1991; Corrected reprint, Cambridge Univ. Press, 1994. MR 92e:15003 MR 95c:15001

[24] V. Istrățescu, Introduction to Linear Operator Theory, Marcel Dekker, New York, 1981. MR 83d:47002

[25] G. James and M. Liebeck, Representations and Characters of Groups, Cambridge Mathematical Textbooks, Cambridge University Press, 1993. MR 94h:20007

[26] A.A. Jafarian and A.R. Sourour, Spectrum preserving linear maps, J. Functional Analysis 66 (1986), 255-261. MR 87m:47011

[27] R. V. Kadison, Isometries of operator algebras, Ann. of Math. 54 (1951), 325-338. MR 13:2569

[28] C.K. Li, The decomposable numerical radius and numerical radius of a compound matrix, Linear Algebra Appl. 76 (1986), 45-58. MR 87g:15037

[29] C.K. Li, Linear operators preserving the numerical radius of matrices, Proc. Amer. Math. Soc. 99 (1987), 601-608. MR 87m:15004

[30] C.K. Li, Matrices with some extremal properties, Linear Algebra Appl. 101 (1988), 255-267. MR 89c:15025

[31] C.K. Li and S. Pierce, Linear operators preserving certain singular matrix sets, Linear and Multilinear Algebra 36 (1993), 19-25. MR 96b:15005

[32] C.K. Li, P. Mehta and L. Rodman, Linear operators preserving the inner and outer c-spectral radius, Linear and Multilinear Algebra 36 (1994), 195-204. MR 96b:15055

[33] C.K. Li and N.K. Tsing, Linear operators preserving the decomposable numerical radius, Linear and Multilinear Algebra 23 (1988), 333-341. MR 90g:15041

[34] C.K. Li and N.K. Tsing, Linear operators preserving the unitarily invariant norms on matrices, Linear and Multilinear Algebra 26 (1990), 119-132. MR 91g:15017

[35] M. Marcus, All linear operators leaving the unitary group invariant, Duke Math. J. 26 (1959), 155-163. MR 21:54 
[36] M. Marcus, Finite Dimensional Multilinear Algebra, Part I, Marcel Dekker, New York, 1973. MR 50:4599

[37] M. Marcus, Finite Dimensional Multilinear Algebra, Part II, Marcel Dekker, New York, 1975. MR 53:5623

[38] M. Marcus and P. Andresen, The numerical radius of exterior powers, Linear Algebra Appl. 16 (1977), 131-151. MR 58:16737

[39] M. Marcus and I. Filippenko, On the unitary invariance of the numerical radius, Pac. J. Math. 75 (1978), 383-395. MR 58:10953

[40] M. Marcus and I. Filippenko, Linear operators preserving the decomposable numerical range, Linear and Multilinear Algebra 7 (1979), 27-36. MR 80d:15028

[41] M. Marcus and B.N. Moyls, Linear transformations on algebras of matrices, Canad. J. Math. 11 (1959), 61-66. MR 20:6432

[42] M. Marcus and M. Sandy, Conditions for the generalized numerical range to be real, Linear Algebra Appl. 71 (1985), 219-239. MR 87a:15036

[43] M. Marcus and B. Wang, Some variations on the numerical range, Linear and Multilinear Algebra 9 (1980), 111-120. MR 82d:47007

[44] F.D. Murnaghan, On the field of values of a square matrix, Proc. Nat. Acad. Sci. USA 18 (1932), 246-248.

[45] R. Merris, Multilinear Algebra, Algebra, Logic and Applications, 8. Gordon and Breach Science Publishers, Amsterdam, 1997. MR 98i:15002

[46] V.J. Pellegrini, Numerical range preserving operators on a Banach algebra, Studia Math. 54 (1975), 143-147. MR 52:8941

[47] S. Pierce et al., A Survey of Linear Preserver Problems, Linear and Multilinear Algebra 33 (1992), 1-130.

[48] V.P. Platonov and D.Z. Doković, Linear preserver problems and algebraic groups. Math. Ann. 303 (1995), no. 1, 165-184. MR 96m:20072

[49] V.P. Platonov and D. Z. Doković, Subgroups of $G L\left(n^{2}, \mathbb{C}\right)$ containing $P S U(n)$, Trans. Amer. Math. Soc. 348 (1996), 141-152. MR 96j:20063

[50] H. Robinson, Quadratic forms on symmetry classes of tensors, Linear and Multilinear Algebra 4 (1977), 233-241. MR 57:359

[51] H. Schneider, Positive operators and an inertial theorem, Numerische Mathematik 7 (1965), 11-17. MR 30:3888

[52] T.Y. Tam, On the generalized $m$ th decomposable numerical radius on symmetry classes of tensors, Linear and Multilinear Algebra 19 (1986), 117-132. MR 87i:15019

[53] T.Y. Tam, Linear operator on matrices: the invariance of the decomposable numerical range, Linear Algebra Appl. 85 (1987), 1-7. MR 88a:15050

[54] T.Y. Tam, Linear operator on matrices: the invariance of the decomposable numerical radius, Linear Algebra Appl. 87 (1987), 147-153. MR 87m:15060

[55] T.Y. Tam, Linear operator on matrices: the invariance of the decomposable numerical range. II, Linear Algebra Appl. 92 (1987), 197-202. MR 88j:15026

[56] W. Watkins, Linear maps and tensor rank, J. Algebra 38 (1976), 75-84. MR 54:12813

[57] H. Weyl, Inequalities between the two kinds of eigenvalues of a linear transformation, Proc. Nat. Acad. Sci. USA 35 (1949), 408-411. MR 11:37d

[58] A. Wintner, Zür Theorie der beschränkten Bilinearformen, Math. Z. 39 (1929), 228-282.

Department of Mathematics, College of William and Mary, P.O. Box 8795, WilliamsBURG, VIRGINIA 23187-8795

E-mail address: ckli@math.wm.edu

Department of Mathematics, University of Toronto, Toronto, Ontario, Canada M5S 3G3 and Institute of Mathematics of The Romanian Academy, 70700 Bucharest, Romania

E-mail address: zaharia@math.toronto.edu 